\title{
A model of ovulatory regulation examining the effects of insulin-mediated testosterone production on ovulatory function
}

\author{
Erica J. Graham ${ }^{\mathrm{a}, *}$, James F. Selgrade ${ }^{\mathrm{b}}$ \\ ${ }^{a}$ Mathematics Department, Bryn Mawr College, Bryn Mawr, Pennsylvania, 19010 \\ ${ }^{b}$ Department of Mathematics and Biomathematics Program, North Carolina State University, Raleigh, North Carolina, 27695
}

\begin{abstract}
Polycystic ovary syndrome (PCOS), a common cause of infertility in women, is often accompanied by abnormal reproductive and metabolic hormone levels. Specifically, androgens such as testosterone are elevated in many PCOS women, and the syndrome itself is frequently associated with insulin resistance, which leads to hyperinsulinemia, i.e., elevated insulin. Although the precise role of insulin in ovulatory function is unclear, its role in ovulatory dysfunction is often linked to the effects of increased ovarian androgen production. We present a mathematical model of the menstrual cycle that incorporates regulation by the pituitary-ovarian axis and mechanisms of ovarian testosterone production. We determine a physiological role for testosterone in the normal ovulatory cycle and study the role of hyperinsulinemia in pathological regulation of the cycle. Model results indicate increased ovulatory disruption with elevated insulin-mediated testosterone production and suggest that variations in the response of ovarian follicles to essential signals can alter the degree to which hyperinsulinemia disrupts the ovulatory cycle. The model also provides insight into the various PCOS phenotypes and the severity of ovulatory dysfunction.
\end{abstract}

Keywords: Polycystic ovary syndrome, steroidogenesis

\section{Introduction}

Polycystic ovary syndrome (PCOS) is a common cause ${ }_{27}^{26}$ 3 of infertility in women. There are three factors that can ${ }_{28}$ lead to PCOS: elevated androgen levels, ovulatory dysfunction, and a polycystic ovary morphology. Of these ${ }^{29}$ factors, more than half of women with PCOS exhibit signs ${ }^{30}$ of hyperandrogenism [1, which may lead to ovulatory dys- ${ }^{31}$ function [2]. The pathogenesis of PCOS is currently un- ${ }^{32}$ known, and because so many women of child-bearing age ${ }^{33}$ are affected, it is important to find the most effective treat- ${ }^{34}$ ments for the syndrome to restore normal ovulatory func- ${ }^{35}$ tion.

Clinical observations of women with PCOS have shown ${ }^{37}$ a frequent association with insulin resistance, i.e. reduced ${ }^{38}$ cellular responses to insulin signaling. Insulin resistance is ${ }^{39}$ most commonly associated with type 2 diabetes, and its ${ }^{40}$ association with PCOS is peculiar. Notably, most women ${ }^{41}$ with PCOS and hyperandrogenism are also hyperinsuline- ${ }_{42}$ mic [1, 3, 4, the latter being a telltale sign of insulin resis- 43 tance. Although the precise role of insulin in ovulatory ${ }_{44}$ function is unclear, its role in ovulatory dysfunction is ${ }_{45}$ often linked to the effects of increased ovarian androgen ${ }_{46}$ production 1]. In addition, treatments of PCOS symp- ${ }_{47}$ toms include the use of insulin sensitizing drugs such as ${ }_{48}$

*Corresponding author. Address: Mathematics Department, $101^{50}$ N. Merion Ave., Bryn Mawr, PA 19010, United States.

Email address: ejgraham@brynmawr.edu (Erica J. Graham) metformin and thiazolidinediones [5, 6]. When successful, these drugs appear to reduce circulating testosterone $(\mathrm{T})$, a common androgen, via a reduction in plasma insulin levels.

The importance of insulin and $\mathrm{T}$ in many PCOS cases is evident. In order to identify their role in ovulatory dysfunction more completely, we look to the dynamics underlying normal ovulation. The menstrual cycle itself is regulated by orchestrated feedback in the hypothalamicpituitary-ovarian axis. Briefly, GnRH neurons in the hypothalamus of the brain are activated to stimulate the production and release of follicle stimulating hormone (FSH) and luteinizing hormone ( $\mathrm{LH}$ ) from gonadotropes in the anterior pituitary 77. Circulating FSH and LH then bind to receptors on ovarian cells to regulate follicle development and production of steroid hormones, namely estradiol $\left(\mathrm{E}_{2}\right)$, progesterone $\left(\mathrm{P}_{4}\right)$, and $\mathrm{T}[8,9$.

$\mathrm{T}$ concentrations in women are significantly lower than those found in men, and accurate measurements are sparse due to a lack of suitably sensitive assays [10, 11. This reduces our ability to characterize $\mathrm{T}$ dynamics fully during the ovulatory cycle. Although $\mathrm{E}_{2}$ and $\mathrm{P}_{4}$ are responsible for much of the feedback to the brain, $\mathrm{T}$ is still thought to play an important role in both follicular development and in tonic (basal) levels of luteinizing hormone (LH). Furthermore, even if $\mathrm{T}$ does not play a significant role in establishing the physiological ovulatory cycle, it is clear that elevated $\mathrm{T}$ can result in dysfunction and infertility. 
It is therefore important, even with limited data, to un- 92 derstand the mechanisms contributing to the behavior of 93 $\mathrm{T}$ throughout the ovulatory cycle.

In this work, we model the menstrual cycle, with special 95 consideration of mechanisms of ovarian steroid production. 96 In Sections 2 and 3 , we develop the mathematical model. 97 In Section 4, we fit the model to clinical data in the lit-98 erature. In Section 5, we present simulation results under 99 normal and pathological circumstances. Finally, in Sec-100 tion 6, we discuss model implications on mechanisms and ${ }_{101}$ the severity of ovulatory dysregulation.

\section{Adaptation of Existing Frameworks: Pituitary} Hormone and Follicle Dynamics

Here we develop models of pituitary regulation, follicle ${ }^{107}$ maturation, and ovarian steroid production. In particular, ${ }^{108}$ we modify and extend existing models of the production ${ }^{109}$ and release of pituitary hormones 12 14 and develop a ${ }^{110}$ new model of stage-dependent follicle maturation based ${ }^{11}$ on 12 15. and introduee a semi-mechanistie model of ${ }^{12}$ ovarian production of $\mathrm{T}, \mathrm{E}_{2}$, and $\mathrm{P}_{4}$. Because our method- ${ }^{113}$ ology incorporates cellular mechanisms of production and our primary focus is the ovaries, we assume implicit feedback effects on and from GnRH neurons. The function of these neurons in response to circulating and pituitary hormones is therefore not modeled within this work.

\subsection{Pituitary regulation}

The dynamics of the pituitary hormones, LH and FSH, are illustrated in Figure 1 following the model of [12. The potential impact of insulin (I) is also depicted, via its effect on T. The state variables in this subsystem include releasable FSH $\left(F S H_{\rho}\right)$, serum FSH $(F S H)$, releasable LH $\left(L H_{\rho}\right)$, and serum LH $(L H)$. We distinguish between releasable and serum quantities of $\mathrm{LH}$ and $\mathrm{FSH}$ to reflect the differential effects of ovarian stimulation and inhibition. For further discussion on this framework, see [12, 14].

Inhibins are another class of hormone produced by the ovaries; they primarily suppress FSH synthesis 7 . There are two major types of inhibin. Inhibin A is the dominant inhibin during the luteal stage, whereas Inhibin B peaks during both the follicular and luteal stages [16. Although both inhibins reduce FSH production, we focus on the effects sustained during the luteal phase, where FSH inhibition is strongest. We do not model inhibin dynamics explicitly; instead, we incorporate suppression of FSH synthesis through luteal stage $(\Lambda)$ dynamics (see Equation $1 \mathrm{a}$ and Section 2.2 Equation 2c and Equation 2d). FSH release into the serum is subsequently promoted by $\mathrm{P}_{4}$ and inhibited by $\mathrm{E}_{2}$. Once in circulation, $\mathrm{FSH}$ decays linearly (see Equation 1b).

Testosterone is an important regulator of LH synthesis by gonadotropes in the pituitary gland. Specifically, T can contribute to a GnRH-mediated increase in LH in both adult female rats 17 . and adolescent girls 18. However, this effect may be reversed with high $\mathrm{T}$ levels in normal, but not PCOS, ovaries [18. The nature of these differential effects is unknown. Therefore, we assume testosterone-dependent LH synthesis simply saturates with maximal rate $v_{0 L}$. It is also hypothesized that hyperandrogenism reduces the ability of $\mathrm{P}_{4}$ to suppress $\mathrm{E}_{2}$-stimulated LH synthesis by modulating the sensitivity of the hypothalamus to $\mathrm{E}_{2}$ and $\mathrm{P}_{4}$ through an unknown mechanism [18, 19. Fur-

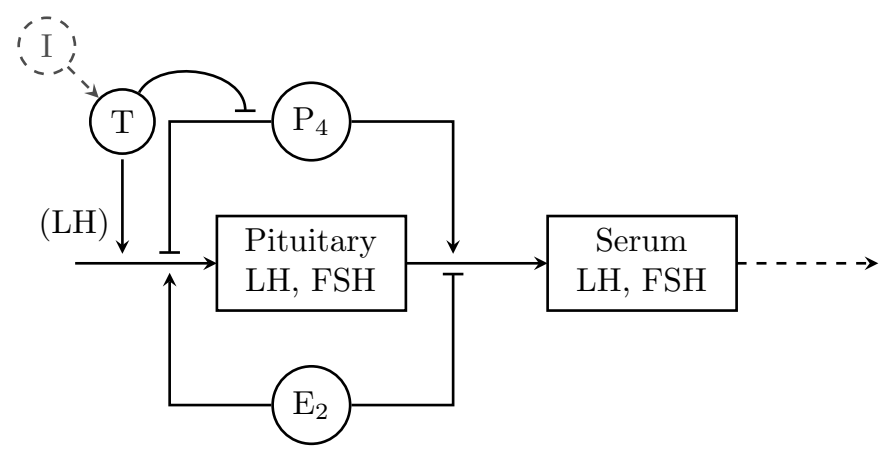

Figure 1. Schematic of pituitary hormone dynamics, as regulated by ovarian steroids $\mathrm{T}, \mathrm{P}_{4}$, and $\mathrm{E}_{2}$. A two-compartment model describes the dynamics of releasable (pituitary) and serum hormones. T serves two functions in the pituitary: It allows for basal LH production, and it prevents inhibition of LH synthesis by $\mathrm{P}_{4} \cdot \mathrm{P}_{4}$ is also important for the release of both $\mathrm{LH}$ and $\mathrm{FSH} . \mathrm{E}_{2}$, on the other hand, inhibits this release into the serum. From the serum, both pituitary hormones experience first-order decay. Insulin (I) may affect these processes via its effect on $\mathrm{T}$ production. $\rightarrow$ denotes stimulation; $-\mid$ denotes inhibition.

$$
\begin{aligned}
& \text { Releasable FSH: } \frac{\mathrm{d} F S H_{\rho}}{\mathrm{d} t}=\frac{v_{F}}{1+c_{F, I} \frac{S \Lambda}{K_{F, I}+S \Lambda}}-k_{F} \frac{1+c_{F, P} P_{4}}{1+c_{F, E} E_{2}^{2}} F S H_{\rho} \\
& \text { Serum FSH: } \quad \frac{\mathrm{d} F S H}{\mathrm{~d} t}=\frac{1}{V} \cdot k_{F} \frac{1+c_{F, P} P_{4}}{1+c_{F, E} E_{2}^{2}} F S H_{\rho}-\delta_{F} F S H \\
& \text { Releasable LH: } \quad \frac{\mathrm{d} L H_{\rho}}{\mathrm{d} t}=\left[v_{0 L} \cdot \frac{T}{K_{L, T}+T}+v_{1 L} \cdot \frac{E_{2}^{n}}{K_{m L}^{n}+E_{2}^{n}}\right] \cdot \frac{1}{1+P_{4} /\left[K_{i L, P}\left(1+c_{L, T} T\right)\right]}-k_{L} \frac{1+c_{L, P} P_{4}}{1+c_{L, E} E_{2}} L H_{\rho} \\
& \text { Serum LH: } \quad \frac{\mathrm{d} L H}{\mathrm{~d} t}=\frac{1}{V} \cdot k_{L} \frac{1+c_{L, P} P_{4}}{1+c_{L, E} E_{2}} L H_{\rho}-\delta_{L} L H
\end{aligned}
$$


ther, this effect appears to be reversed, with normal hy-155 pothalamic GnRH pulse generator sensitivity, upon treat-156 ment with an antiandrogen 20 . We assume this inhibition ${ }_{15}$ also exists under normal circumstances and hence use the ${ }_{158}$ expression $1+c_{L, T} T$ to model this effect. $\mathrm{P}_{4}$ and $\mathrm{E}_{2}$ have ${ }_{159}$ similarly opposing effects in LH release as in FSH release ${ }_{160}$ (see Equation 1c).

\subsection{Follicle dynamics}

The ovulatory cycle can be broadly categorized into16 three major phases. The follicular phase is a time of ${ }_{165}$ growth and development, characterized by selection of a166 dominant follicle for ovulation [21, 22]. The ovulatory ${ }_{167}$ phase is precipitated by a surge in serum LH, which stimulates the rupture of the dominant follicle and ovum release. The luteal phase is characterized by the development and subsequent regression of the corpus luteum, wherein the ruptured follicle undergoes extensive remodeling, becomes highly vascularized, and produces large amounts of $\mathrm{P}_{4}$ [23].

Previous models have accounted for as many as 12 stages of follicle growth and maturation $12-15$. To reduce the complexity of the present model yet capture essential dynamics, we consider only the three major stages of ovarian development: follicular, ovulatory, and luteal. These theoretical stages reflect a contribution to the ovarian mass (in micrograms) at any given time. We remark that Chen and Ward 15 also consider a three-stage reduction when modeling the menstrual cycle.

Figure 2 illustrates the regulation of follicle development by FSH and LH. Androgens stimulate the growth of small follicles, during the early stages of development [24, 25]. It has been shown that androgens, in particular, increase FSH receptor expression in follicular cells [26, 27]. We therefore assume that $\mathrm{T}$ is the androgen responsible for increasing baseline sensitivity to FSH. Once mature enough, follicles increase their mass while in follicular phase $\Phi$ in response to growth signals from FSH (see Equation 2a).

LH is the primary ovulation signal, responsible for the transition from stage $\Phi$ to ovulatory stage $\Omega$, which may be affected by insulin (discussed in Section 5.3). FSH increases the number of LH receptors during the mid-tolate follicular stage, to prepare follicles for luteinization
28, 29. Luteinization is the process through which cells in the pre-ovulatory follicle terminally differentiate into luteal cells, wherein they undergo hypertrophy and accumulate lipids [30]. Follicle sensitivity to LH is therefore mediated by the presence of FSH, resulting in an FSHinduced leftward shift of the dose-response curve of LHdependent follicular differentiation (see Equation 2a).

Once in the ovulatory phase, $\Omega$, follicle rupture leads to the formation of the corpus luteum and a transition to the luteal stage, $\Lambda$ (Equation 2b). This is an LH-dependent process, which allows the corpus luteum to both develop and mature [31]. During this stage, LH levels are much lower than surge levels. Still, it is evident LH is required

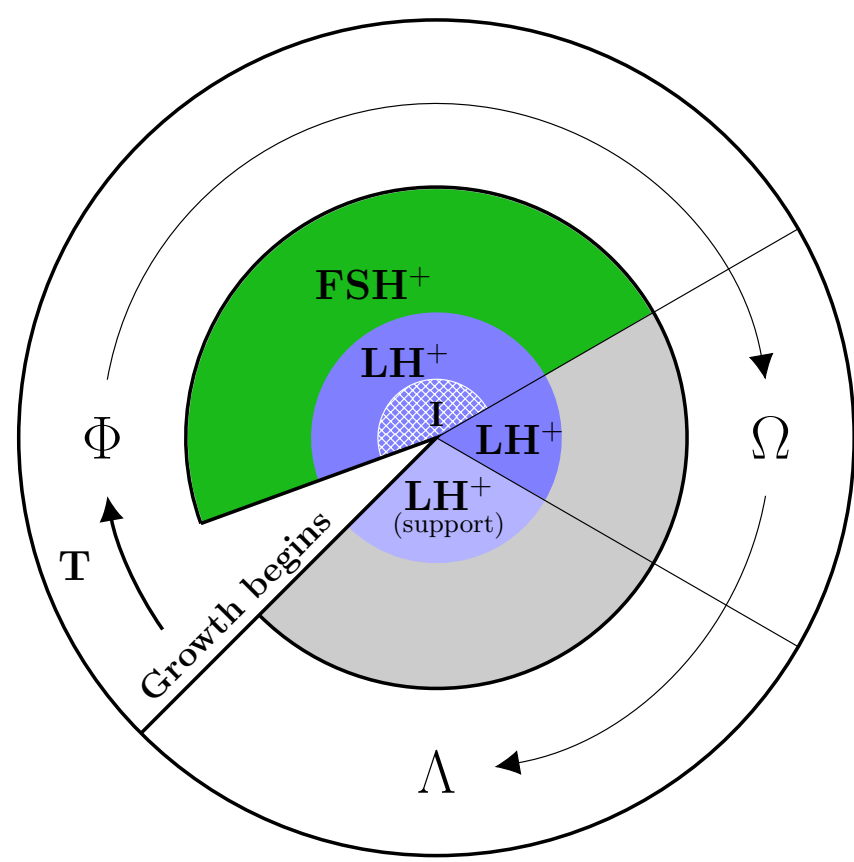

Figure 2. Schematic of follicular dynamics subsystem. $\mathrm{T}$ is responsible for early growth/maturation of immature follicles. It also increases the sensitivity of these follicles to FSH. Later, these follicles respond to LH, progressing from follicular stage $\Phi$ to ovulatory stage $\Omega$. $\mathrm{LH}$ is further required, in a supporting role, for the transition to the luteal stage $\Lambda$ and subsequent maintenance of the corpus luteum. Insulin (I) may also play a role in the $\Phi$-to- $\Omega$ transition.

$$
\begin{array}{ll}
\text { Follicular phase: } & \frac{\mathrm{d} \Phi}{\mathrm{d} t}=f_{0} \cdot \frac{T}{T_{0}}+\left[\frac{f_{1} F S H^{2}}{\left(\frac{h_{1}}{1+T / T_{0}}\right)^{2}+F S H^{2}}-\frac{f_{2} L H^{2}}{\left(\frac{h_{2}}{1+c_{\Phi, F} F S}\right)^{2}+L H^{2}}\right] \cdot \Phi \\
\text { Ovulatory phase: } \frac{\mathrm{d} \Omega}{\mathrm{d} t}=\frac{f_{2} L H^{2}}{\left(\frac{h_{2}}{1+c_{\Phi, F} F S H}\right)^{2}+L H^{2}} \cdot \Phi-w S \Omega \\
\text { Luteal phase: } & \frac{\mathrm{d} \Lambda}{\mathrm{d} t}=w S \Omega-l(1-S) \Lambda \\
\text { LH Support: } & \frac{\mathrm{d} S}{\mathrm{~d} t}=\frac{\hat{s} L H^{m}}{h_{s}^{m}+L H^{m}} \cdot(1-S)-\delta_{s} S
\end{array}
$$


to sustain the corpus luteum, as experimental depletion of 202 post-ovulatory LH results in insufficient luteal growth and 203 maturation 31, 32.

We introduce the state variable $S$ to represent LH sup-205 port. During the luteal stage, support is extremely sen-206 sitive to LH, even at very low levels. Under these cir-207 cumstances, $S$ may approach a maximal value of 1 . In the 208 absence of LH, support decays, and $S$ may tend toward 209 0 (see Equation $2 \mathrm{~d}$ ). Therefore, $0 \leqslant S(t) \leqslant 1$ for all $t$. We 210 regard LH support as a permissive state during which nor-211 mal luteal activity, specifically luteal growth and hormone212 production, can occur. Terms involving the luteal stage 213 are multiplied by $S$ to indicate a lack of activity in the 214 absence of sufficient functional support by LH.

The signals that promote luteal regression, or luteolysis, 216 in humans are largely unknown and widely hypothesized 217 30, 32. However, because LH is important for the devel-218 opment and maintenance of the corpus luteum, we assume219 that LH support also prevents premature luteolysis. Thus,220 the rate of luteal regression is taken to be proportional to221 $(1-S) \Lambda$ (see Equation 2c).

\section{Model Development: Ovarian Steroidogenesis ${ }^{224}$}

Here we introduce a semimechanistic model of ovarian ${ }_{22}$ steroidogenesis that incorporates the influence of hyperin-227 sulinemia. We develop the model in three parts; the first ${ }_{228}$ presents a preliminary model of intracellular androgen pro-229 duction, the second is a model reduction to testosterone ${ }_{230}$ dynamics, and the third extends the reduced model to in-231 corporate follicle dynamics.

Production of any of the ovarian steroids, such as $\mathrm{T}_{, 233}$ $\mathrm{E}_{2}$, and $\mathrm{P}_{4}$, results from a series of enzymatic conversions234 of cholesterol and its derivatives. A two-cell hypothesis 235 provides the prevailing explanation of the origins of the 236 major ovarian steroids. The two cell types within follicles 237 are theca cells and granulosa cells (see Figure 3). In the hypothesis, theca cells are responsible for the production of androgens, especially androstenedione (A), dihydrotestosterone, and T. Granulosa cells, on the other hand, are responsible for generating $\mathrm{E}_{2}$ and most luteal stage $\mathrm{P}_{4}$ [32].

Careful consideration of intracellular mechanisms of androgen production in follicles supports a mechanistic role for insulin. Insulin resistance, or impaired insulin signaling, sets the stage for many insulin-mediated defects in the ovulatory cycle. Unlike most tissues in the body, ovarian insulin receptors are predominately regulated by steroids and gonadotropins. Therefore, the ovaries tend to remain sensitive to insulin at the receptor level, even in the midst of systemic insulin resistance 33, 34. In PCOS, it is unclear what post-receptor defects occur in ovarian insulin signaling. However, it has been proposed that theca cells are actually hypersensitive to insulin signals, resulting in exaggerated androgen synthesis and secretion relative to $\mathrm{E}_{2}$ and $\mathrm{P}_{4}$ [35, 36]. Further, through its receptor on ovarian granulosa and theca cells, insulin can act as a co-gonadotropin with LH to increase the expression of steroidogenic enzymes, including androgens [33, 37.

\subsection{Preliminary model of ovarian androgen production}

We develop the steroidogenesis model under several major assumptions. First, cholesterol, as the primary precursor to all ovarian steroids, is relatively abundant in theca and granulosa cells. Second, the layers of theca cells in a given follicle function uniformly to respond to $\mathrm{LH}$ and to synthesize androgens. Finally, the primary effect of elevated insulin is augmented LH-mediated steroidogenic enzyme production within theca cells.

For simplicity, we also begin the steroidogenic cascade with A production. This allows us to focus on the effects of insulin and LH at the level of CYP17 production, which permits the eventual conversion of pregnenolone into androgens [38]. Although several androgens are produced by

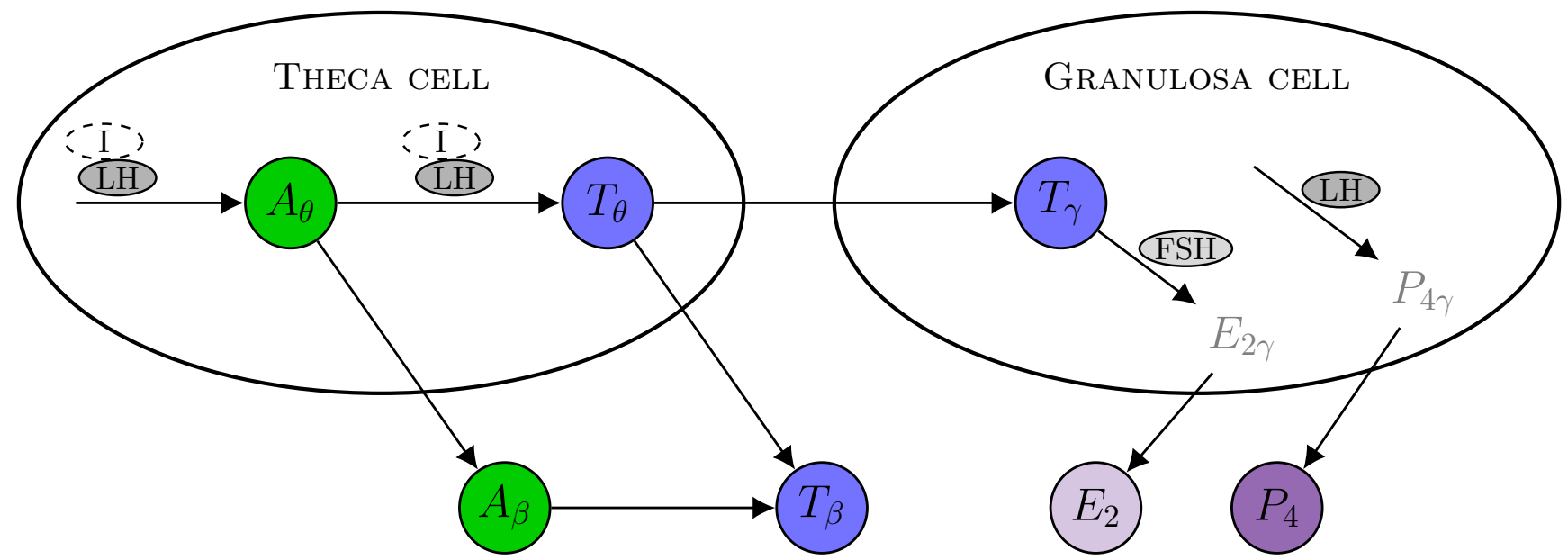

Figure 3. Ovarian steroidogenesis model schematic. The two-cell theory of steroid production is taken into account through quantities residing in both theca and granulosa cells. I, LH, and FSH denote processes that are dependent on insulin, LH, and FSH, respectively. The preliminary model takes into account synthesis and secretion of androgens (androstenedione and testosterone) and estradiol. Gray text denotes quantities not explicitly modeled in this subsystem, i.e. intracellular $\mathrm{E}_{2}\left(E_{2 \gamma}\right)$ and $\mathrm{P}_{4}\left(P_{4 \gamma}\right)$. 
Theca cell A: $\quad \frac{\mathrm{d} A_{\theta}}{\mathrm{d} t}=k_{0} \mathcal{G}_{1}(I) \cdot H\left(L H, \hat{h}_{1}, 1\right)-k_{1} \mathcal{G}_{2}(I) \cdot H\left(L H, \hat{h}_{2}, 1\right) \cdot A_{\theta}-k_{1}^{\prime} A_{\theta}$

Theca cell T: $\quad \frac{\mathrm{d} T_{\theta}}{\mathrm{d} t}=k_{1} \mathcal{G}_{2}(I) \cdot H\left(L H, \hat{h}_{2}, 1\right) \cdot A_{\theta}-k_{2}^{\prime} T_{\theta}$

Granulosa cell T: $\frac{\mathrm{d} T_{\gamma}}{\mathrm{d} t}=q k_{2}^{\prime} T_{\theta}-k_{2} H\left(F S H, h_{3}, 1\right) T_{\gamma}$

Circulating A: $\quad \frac{\mathrm{d} A_{\beta}}{\mathrm{d} t}=k_{1}^{\prime} A_{\theta}+k_{4}-\delta_{A} A_{\beta}-k_{3} A_{\beta}$

Circulating T:

$$
\frac{\mathrm{d} T_{\beta}}{\mathrm{d} t}=k_{3} A_{\beta}+k_{5}-\delta_{T} T_{\beta}+(1-q) k_{2}^{\prime} T_{\theta}
$$

where $H(x, h, n)=\frac{x^{n}}{x^{n}+h^{n}}$ is a Hill function with Hill coefficient $n$.

theca cells, we take A to be the major precursor and $\mathrm{T}$ the 279 major product.

The state variables representing androgens in the intracellular system are theca cell $\mathrm{A}\left(A_{\theta}\right)$, circulating $\mathrm{A}\left(A_{\beta}\right)$, theca cell $\mathrm{T}\left(T_{\theta}\right)$, granulosa cell $\mathrm{T}\left(T_{\gamma}\right)$, and circulating $\mathrm{T}$ $\left(T_{\beta}\right)$. The dynamics considered in this subsystem, along with their dependence on insulin, FSH, and LH, are illustrated in Figure 3 To focus on the effects of insulinmediated ovarian testosterone production in particular, we derive a model only for androgen production. Dynamics for $\mathrm{E}_{2}$ and $\mathrm{P}_{4}$ will be considered alongside follicle dynamics in Section 3.3

In the theca cell model, A is created downstream of intracellular cholesterol through enzyme activity promoted by LH and enhanced by elevated insulin (see Equation 3a). The concentration of A decreases either upon release into circulation or intracellular conversion into T. For simplicity, we assume that $\mathrm{T}$ is the only androgen to be aromatized, i.e. converted into $\mathrm{E}_{2}$, within granulosa cells.

We define arbitrary functions $\mathcal{G}_{1}=\mathcal{G}_{1}(I)$ and $\mathcal{G}_{2}=$ $\mathcal{G}_{2}(I)$ to represent the influence of insulin, $I$, in enzyme production for conversion from precursor steroids to $\mathrm{A}$ and from A into T, respectively. We assume insulin can augment cellular responses to LH in this capacity, so $\mathcal{G}_{1}$ and $\mathcal{G}_{2}$ are increasing functions of $I$. At basal insulin levels, we take $\mathcal{G}_{1}=\mathcal{G}_{2}=1$.

We model theca cell $\mathrm{T}$ to be created from its precursor A and subsequently secreted, with a fraction $q$ entering neighboring granulosa cells (see Equation $3 \mathrm{~b}$ ) and the remainder released directly into the circulation. Proximity between theca and granulosa cells is assumed sufficiently ${ }^{28}$ small to accommodate rapid transfer between the two cell types. Granulosa cell T transferred from the theca cell is then aromatized via FSH signaling (see Equation 3c).

In Equation 3d we assume circulating $\mathrm{A}\left(A_{\beta}\right)$ comes directly from theca cells or is produced by the adrenal gland at a constant rate. Once in the bloodstream, A may be converted peripherally into T. This conversion contributes to the circulating $\mathrm{T}$ concentration, along with direct theca cell secretion and constant basal production (see Equation 282 $3 \mathrm{e}$.

\subsection{Reduction of preliminary model}

As defined, Equations (3a)-(3e) represent the dynamics of intracellular androgen concentrations from individual cellular masses. Here we reduce the model to essential dynamics captured by variables $T_{\gamma}$ and $T_{\beta}$, under the assumptions that theca cell steroid production is balanced with release and that $\mathrm{A}$, in its various forms, also experiences zero net change. To construct the simplified model for testosterone dynamics, we use these assumptions to solve for $A_{\theta}, T_{\theta}$, and $A_{\beta}$. Then the reduced model can be written as

$$
\begin{aligned}
\frac{\mathrm{d} T_{\gamma}}{\mathrm{d} t} & =q k_{0} Q_{1}-k_{2} H\left(F S H, h_{3}, 1\right) T_{\gamma}, \\
\frac{\mathrm{d} T_{\beta}}{\mathrm{d} t} & =k_{5}-\delta_{T} T_{\beta}+\frac{k_{3} k_{4}}{k_{3}+\delta_{A}}+\frac{k_{1}^{\prime} k_{0}}{k_{1}\left(k_{3}+\delta_{A}\right)} \cdot Q_{2} \\
& +(1-q) k_{0} Q_{1},
\end{aligned}
$$

where $Q_{1}=\mathcal{G}_{1}(I) \mathcal{G}_{2}(I) \cdot L H^{2} / \mathcal{D}, Q_{2}=\left[\mathcal{G}_{1}(I) L H^{2}+\right.$ $\left.\mathcal{G}_{1}(I) L H \cdot \hat{h}_{2}\right] / \mathcal{D}$,

$$
\begin{aligned}
\mathcal{D}= & L H^{2}\left[\mathcal{G}_{2}(I)+\frac{k_{1}^{\prime}}{k_{1}}\right]+ \\
& +L H\left[\mathcal{G}_{2}(I) \hat{h}_{1}+\frac{k_{1}^{\prime}}{k_{1}} \cdot\left(\hat{h}_{1}+\hat{h}_{2}\right)\right]+\frac{k_{1}^{\prime}}{k_{1}} \cdot \hat{h}_{1} \hat{h}_{2} \\
= & L H^{2}\left[\mathcal{G}_{2}(I)+A\right]+L H\left[\mathcal{G}_{2}(I) B+A \cdot(B+C)\right]+ \\
& +A \cdot B \cdot C,
\end{aligned}
$$

and where the new parameters are defined by $A=k_{1}^{\prime} / k_{1}$, $B=\hat{h}_{1}$, and $C=\hat{h}_{2}$.

We further define

$$
\begin{aligned}
& F_{1}=F_{1}(L H, I)=L H^{2} / \mathcal{D} \text { and } \\
& F_{2}=F_{2}(L H, I)=L H / \mathcal{D},
\end{aligned}
$$

so that

$$
\begin{aligned}
Q_{1} & =\mathcal{G}_{1}(I) \cdot \mathcal{G}_{2}(I) \cdot F_{1} \quad \text { and } \\
Q_{2} & =\mathcal{G}_{1}(I)\left[F_{1}+\hat{h}_{2} F_{2}\right] .
\end{aligned}
$$

The functions $F_{1}$ and $F_{2}$ reflect the contributions of theca cells and the periphery toward overall concentrations of 


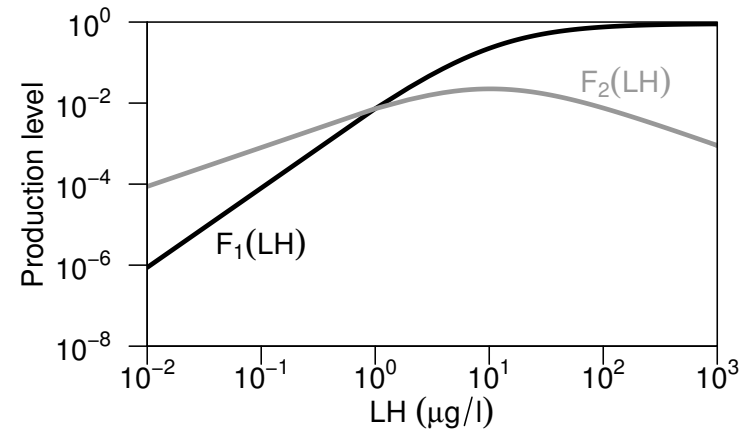

(a)

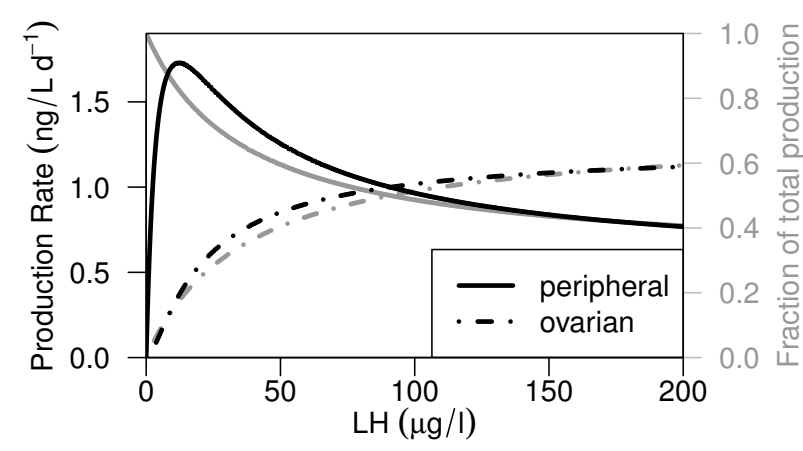

(b)

Figure 4. Functions used to determine ovarian and peripheral testos- 323 terone production. (a) Functions $F_{1}$ and $F_{2}$. (b) Peripheral and 324 ovarian production rates.

T. $F_{1}$ is a saturating dose-response curve of $\mathrm{T}$ production ${ }^{32}$ via LH. $F_{2}$ is an inverted U-shaped dose-response curve, ${ }^{328}$ wherein both low and high levels of LH reduce T produc- ${ }^{329}$ tion, and maximal stimulation occurs at an intermediate ${ }^{330}$ level of LH (see Figure 44a)). Using $F_{1}$ and $F_{2}$, we classify ${ }^{331}$ two forms for generalized $\mathrm{T}$ production:

1. ovarian $\mathrm{T}$ production rate proportional to $F_{1}$

2. peripheral $\mathrm{T}$ production rate proportional to $F_{1}+\hat{h}_{2} F_{2}$.

Under basal conditions, about $60 \%$ of circulating $\mathrm{T}$ com from peripheral conversion. In contrast, the ovaries compose the major source of $\mathrm{T}$ when LH is elevated 1, 29, 39.3 .34 Figure 4 b illustrates this general notion given $F_{1}$ and $F_{2} \cdot 34$

To complete the reduced model of intracellular testos- ${ }_{342}$ terone production, we make the following substitutions: ${ }_{343}$ $t_{g 1}=q k_{0}, t_{g 2}=k_{2}, t_{0}=k_{5}+\frac{k_{3} k_{4}}{k_{3}+\delta_{A}}, t_{1}=\frac{k_{1}^{\prime} k_{0}}{k_{1}\left(k_{3}+\delta_{A}\right)}, 344$ $t_{2}=(1-q) k_{0}$, and $c_{T, F_{2}}=\hat{h}_{2}$. This gives

$$
\begin{aligned}
\frac{\mathrm{d} T_{\gamma}}{\mathrm{d} t}= & t_{g 1} \mathcal{G}_{1} \cdot \mathcal{G}_{2} \cdot F_{1}-t_{g 2} H\left(F S H, h_{3}, 1\right) T_{\gamma}, \\
\frac{\mathrm{d} T_{\beta}}{\mathrm{d} t}= & t_{0}-\delta_{T} T_{\beta}+t_{1} \mathcal{G}_{1}\left[F_{1}+c_{T, F_{2}} F_{2}\right]+ \\
& +t_{2} \mathcal{G}_{1} \cdot \mathcal{G}_{2} \cdot F_{1} .
\end{aligned}
$$
basal insulin level, so after setting $\mathcal{G}_{1}=\mathcal{G}_{2}=1$, we can ${ }_{354}$ redefine $\mathcal{D}=\kappa_{1} L H^{2}+\kappa_{2} L H+\kappa_{3}$, with $\kappa_{1}=1+A$, $\kappa_{2}=B+A(B+C)$, and $\kappa_{3}=A B C$. Note that $C=c_{T, F_{2}}$. After estimating $\kappa_{1}$ and $\kappa_{2}$, the parameters $A, B, C$, and $\kappa_{3}$ can be obtained by solving a nonlinear system of equations and imposing a uniqueness restriction on the solution. By deriving these four parameters, we reduce the number of unknown parameters in the expression $\mathcal{D}$ from three to two.

Finally, to incorporate insulin-mediated effects beyond baseline, we assume that each insulin-dependent term results in an augmented response modeled by a percentage increase in the LH-dependent effect. To that end, we take $\mathcal{G}_{1}=1+\alpha$, where $\alpha$ reflects the degree of insulin influence and $\alpha=0$ at baseline. For simplicity, we set $\mathcal{G}_{2}=1$, noting that this simplification shifts our focus to the upstream effects of insulin, at the level of androgen-producing enzyme CYP17 $(17,20)$ lyase production. With this modification, $F_{1}$ and $F_{2}$ are now insulin-independent functions. Nevertheless, we do not completely remove $\mathcal{G}_{2}$ from the model to allow for the possibility of downstream insulin-induced effects.

\subsection{Complete model of ovarian steroidogenesis subject to follicular dynamics}

With Subsystem 4, we incorporate the effects of elevated insulin on testosterone production in a mechanistic way. We complete the model of ovarian steroid production by subjecting intracellular steroid dynamics to the behavior of the ovarian mass. The state variables in the final steroidogenesis subsystem are serum $\mathrm{T}(T)$, intermediate $\mathrm{T}\left(T_{\gamma}\right)$, serum $\mathrm{E}_{2}\left(E_{2}\right)$ and serum $\mathrm{P}_{4}\left(P_{4}\right)$.

Follicles that have exited the growth arrest stage are equipped with machinery to produce $\mathrm{T}$; we account for this with the product of intracellular production and the ovarian stages (see Equation 5a). In addition, less mature follicles than those in stage $\Phi$ are able to produce testosterone [38]. Therefore, we also assume there is a contribution from the overall ovarian mass not captured within $\Phi+\Omega+\Lambda$.

Unlike the other steroid state variables, $T_{\gamma}$ reflects a concentration per unit ovarian mass (see Equation 5b). Intermediate $\mathrm{T}$, whose production depends on a stimulatory response to $L H$ via $F_{1}$, enters the granulosa cell specifically for FSH-dependent conversion into $\mathrm{E}_{2}$. We assume $\mathrm{E}_{2}$, upon production within granulosa cells, is immediately released into circulation and that this production results only from follicles in the follicular and luteal phases. Any extra-ovarian $\mathrm{E}_{2}$ is produced peripherally by the adrenal gland at a constant rate. We assume $\mathrm{E}_{2}$ experiences firstorder clearance from serum (see Equation $5 \mathrm{c}$ ).

The equation for $\mathrm{P}_{4}$ dynamics (Equation $5 \mathrm{~d}$ ) follows a similar pattern to that of $\mathrm{E}_{2}$. Basal production of $\mathrm{P}_{4}$ outside of the ovaries is negligible [40; we therefore omit this from the model. In addition, although $\mathrm{P}_{4}$ is produced upstream of $\mathrm{T}$, we assume theca cells contribute a negligible amount to the serum $\mathrm{P}_{4}$ level, since most $\mathrm{P}_{4}$ in theca cells is actually converted into A [41. We further assume 


$$
\begin{array}{ll}
\text { Serum T: } & \frac{\mathrm{d} T}{\mathrm{~d} t}=t_{0}-\delta_{T} T+\left[t_{1} \mathcal{G}_{1}\left(F_{1}+c_{T, F_{2}} F_{2}\right)+t_{2} \mathcal{G}_{1} \mathcal{G}_{2} F_{1}\right] \cdot\left[\Phi+\tau_{1} \Omega+\tau_{2} S \Lambda+\tau_{3}\left(1-\frac{\Phi+\Omega+\Lambda}{\Psi}\right)\right] \\
\text { Intermediate } \mathrm{T}: & \frac{\mathrm{d} T_{\gamma}}{\mathrm{d} t}=t_{g 1} \mathcal{G}_{1} \mathcal{G}_{2} F_{1}-\frac{t_{g 2} F S H}{h_{3}+F S H} T_{\gamma} \\
\text { Serum } \mathrm{E}_{2}: & \frac{\mathrm{d} E_{2}}{\mathrm{~d} t}=e_{0}-\delta_{E} E_{2}+\frac{t_{g 2} F S H}{h_{3}+F S H} T_{\gamma} \cdot[\Phi+\eta \Lambda S] \\
\text { Serum } \mathrm{P}_{4}: & \frac{\mathrm{d} P_{4}}{\mathrm{~d} t}=-\delta_{P} P_{4}+\frac{p L H}{L H+h_{p}} \cdot \Lambda S
\end{array}
$$

that relevant quantities of $\mathrm{P}_{4}$ are only produced by the 395 luteal phase in an LH-dependent process. As with the 396 other steroids, we assume first-order clearance. Finally,397 $\mathrm{P}_{4}$ is assumed to be released into circulation immediately 398 upon production within granulosa cells.

\section{Methods}

In order to verify the physiological behavior of the cur- ${ }_{402}$ rent model, we make use of data in the literature and $\mathrm{pa}^{-}{ }_{403}$ rameter estimation tools. Here we discuss the methodology used to generate model results.

\subsection{Comparative data}

Currently, there are no studies with published data of women with daily measurements over an entire ovulatory ${ }^{407}$ cycle for each of the five major hormones (LH, FSH, $\mathrm{E}_{2}, 408$ $\mathrm{P}_{4}$, and $\mathrm{T}$ ) considered in the current model. We therefore 409 combine two sets of published data to verify qualitative 410 dynamics. We use FSH, LH, $\mathrm{E}_{2}$, and $\mathrm{P}_{4}$ data from $422_{411}$ and $\mathrm{T}$ data from 10 for comparison.

Reported average values for each state variable were ap-413 proximated digitally using the digitize package written ${ }_{414}$ for MATLAB 43. The T data used reflects a smoothed 415 average reported by Keefe and colleagues 10. All data416 are converted to SI units where applicable.

Data reported in 42 reflect a presumed 31-day cycle,418 whereas a 28-day cycle is reflected by the $\mathrm{T}$ data in [10.419 To overcome this discrepancy, we first extrapolate the $T_{420}$ data to a 31-day cycle, assuming the peak of the data cor-421 responds to the same day of the preovulatory LH surge 422 reported in 42. In all results reported here, day 15 cor-423 responds to the mid-cycle LH surge in a normal ovulatory 424 cycle.

\subsection{Parameter estimation}

The final model consists of the 12 ordinary differen-428 tial equations listed in systems (1), (2), and (5) with 40 unknown parameters. In order to achieve good qualita-429 tive agreement between model and data, we optimize the4зо parameters of the model using the Nelder-Mead simplex 431 method in MATLAB with weighted least squares. Data $d_{432}$ for a given state variable were given a baseline weight of ${ }_{433}$ $w_{d}=1 / \operatorname{Var}(d)$, where $\operatorname{Var}(d)$ is the variance of the data434 over two cycles, i.e., a 62-day period. Secondary weights were used to ensure that essential events within the cycle, such as the preovulatory LH surge and corpus luteum formation, are captured by the model. The complete list of estimated and fixed parameters, along with initial conditions for state variables, used in the model appear in Table A.1 in AppendixA.

\section{Model Behavior and Investigation of Insulin- Mediated Effects}

We present numerical simulation results of the model, under both normal $(\alpha=0)$ and pathological $(\alpha>0)$ conditions.

\subsection{Dynamics during a normal ovulatory cycle}

Figure 5 shows the results of parameter estimation and the model fit to the data, along with the behavior of the three ovarian stages and the LH support variable, $S$, over time. Although we limit the representative behavior to two cycles, we note that these dynamics reflect stable periodic oscillations with a cycle length of 30.9 days. There is good qualitative agreement between the model and the data. The most significant discrepancy occurs with the variable $\mathrm{T}$, which we attribute to the use of two different data sets. Other substantial differences from the data we attribute to the simplifying assumptions made in the follicular growth subsystem, where we dramatically reduce the number of follicular growth stages. In addition, we have omitted delayed feedback mechanisms, which can provide better agreement with the data 13, 14, 44, 45.

As a novel entity in modeling the ovulatory cycle, $S$ dynamics indicate the support given to luteal growth and function. The support level plateaus at a maximal value once LH concentrations exceed a certain threshold. Support declines with reductions in LH. A loss of $S$ initiates a more rapid decline in $\Lambda$.

\subsection{Simulated hyperinsulinemia}

We can simulate elevations in insulin by varying the parameter $\alpha$ introduced in Section 3.2 . Figure 6 shows the model results for selected values of $0 \leqslant \alpha \leqslant 5$, demonstrating the changes in dynamics for varying levels of feedback. Results are presented with the first LH surge in 

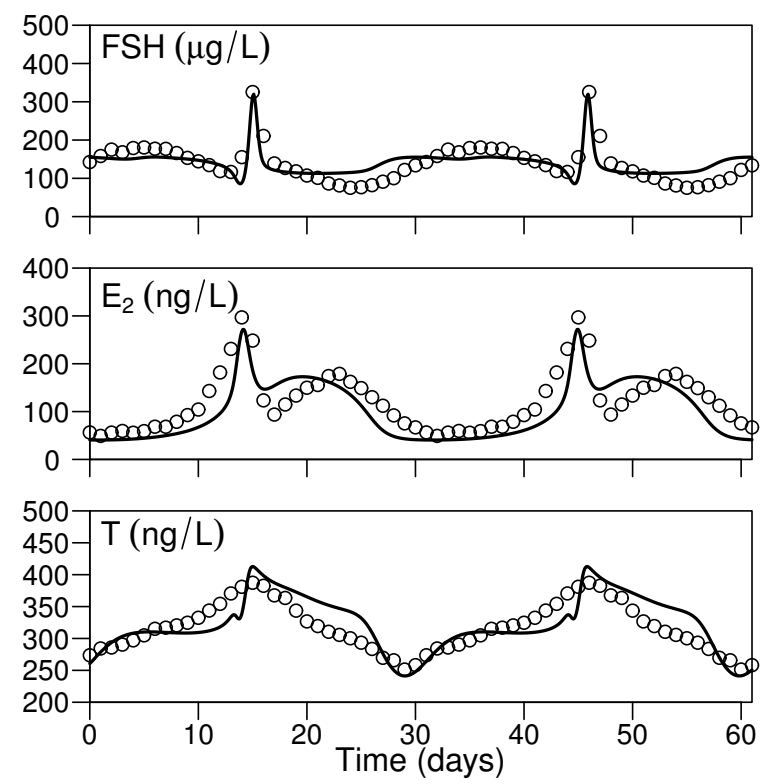
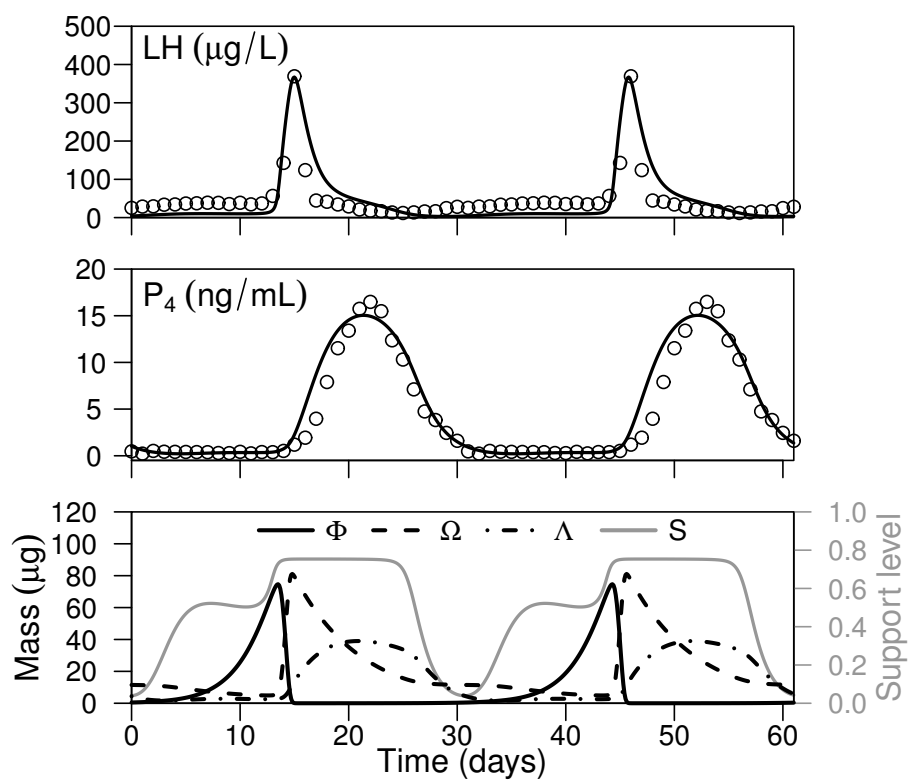

Figure 5. Numerical optimization and simulation results. Comparison to data in 42 (FSH, LH, E $\left.2, \mathrm{P}_{4}\right)$ and 10 ] ( $\left.\mathrm{T}\right)$. Lower right panel: simulated ovarian and LH support trajectories for best fit parameters. Results show a 30.9-day cycle, which is repeated for 2 cycles.

each case placed at day 15 for direct comparison. Over-467 all $\mathrm{T}$ levels rise in a fairly linear fashion with increased $\alpha .468$ LH levels are elevated, particularly at the pre-ovulatory ${ }_{469}$ surge and the luteal stage. Peak LH levels increase for 470 small $\alpha$ and less so with larger $\alpha$. This is due to satura- ${ }_{471}$ tion of basal LH synthesis in the presence of high $\mathrm{T}$. $\mathrm{On}_{472}$ the hand, Peak FSH concentrations decline, whereas 473 luteal stage FSH levels are either increased or the same as 474 the baseline case. This suggests an increased LH:FSH ratio475 with increasing testosterone levels. There is also a slight 476 reduction in cycle length as $\alpha$ increases. This is likely due ${ }_{477}$ to feedback from elevated LH, as follicles in stage $\Phi$ are $_{478}$ prompted to progress to stage $\Omega$ more strongly.

$\mathrm{E}_{2}$ is generally increased with $\alpha$ except at its peak, which ${ }_{480}$ occurs just prior to the pre-ovulatory surge. $\mathrm{E}_{2}$ dynamics481 are determined by increased $T_{\gamma}$ and reduced follicle growth ${ }_{482}$ (results not shown). The $\mathrm{E}_{2}$ peak corresponds to the dip483 in FSH prior to its pre-ovulatory spike, whereas increased ${ }_{484}$ $\mathrm{E}_{2}$ at ovulation corresponds to reduced peak FSH levels. ${ }_{485}$ Notably, higher $\alpha$ corresponds to a reduced $\mathrm{P}_{4}$ ampli-486 tude. This is the result of reduced follicle growth $\operatorname{and}_{487}$ excess LH. Further reductions in $\mathrm{P}_{4}$ (peak less than $3_{488}$ $\mathrm{ng} / \mathrm{mL}$ ) would likely indicate anovulation, as described $\mathrm{in}_{489}$ 46. Collectively, the model produces elevated LH and $\mathrm{P}_{4490}$ deficiency in the presence of insulin-mediated hyperandro-491 genism.

\subsection{Hyperinsulinemia with premature luteinization}

A classic characteristic of PCOS is the arrested growth ${ }_{496}$ of follicles [47, which prevents the preovulatory LH surge.497 In the previous section, model exploration with simulated 498 hyperinsulinemia yields a periodic LH surge with possi-499 bly normal ovulatory cycles for lower $\alpha$ values. With the500 exception of a possible deficiency in $\mathrm{P}_{4}$, no other obvious dysfunction occurs.

We therefore explore an additional mechanism of ovulatory disruption hypothesized to result from hyperinsulinemia. Premature luteinization (PL) is the process through which follicles become responsive to $\mathrm{LH}$ earlier in their maturation. Given the nature of the ovulatory cycle, PL occurs in small follicles, and follicles from individuals with PCOS may be subject to premature luteinization caused by hyperinsulinemia $2,20,48$.

Mathematically, we simulate PL with an insulindependent increase in sensitivity to LH signals during the follicular-to-ovulatory transition. For simplicity, we take $\alpha$ to denote the overall degree of insulin influence in the ovaries. The affected model parameter is $h_{2}$, where increased sensitivity to LH is modeled as $h_{2} /(1+\alpha)$. Therefore, an increased insulin-mediated effect $(\alpha>0)$ results in a leftward shift of the LH dose-response curve in Equations $2 \mathrm{a}$ and $2 \mathrm{~b}$

Figure 7 shows the time courses of selected state variables for $\alpha=0,1,2,3,4$, and 5 . In the presence of PL, increases in $\mathrm{T}$ result in consistent reductions in the preovulatory LH surge until it is completely absent. Because the LH surge is highly variable under PL, the peak of stage $\Omega$-pronounced for every $\alpha$-is used to synchronize initial cycles. Where applicable, the first LH surge is shown at day 15. For most cases in which the LH surge is absent, e.g. $\alpha=4$, the $\mathrm{LH}$ achieves a maximum value during the height of the luteal stage, which also corresponds to the rise in $\mathrm{P}_{4}$. As ovulation becomes more dysfunctional, the range of FSH decreases. The system approaches a steady state when $\alpha=5$. These results indicate that PL exacerbates ovulatory dysfunction and, with elevated T, can eventually eliminate ovulation altogether. 

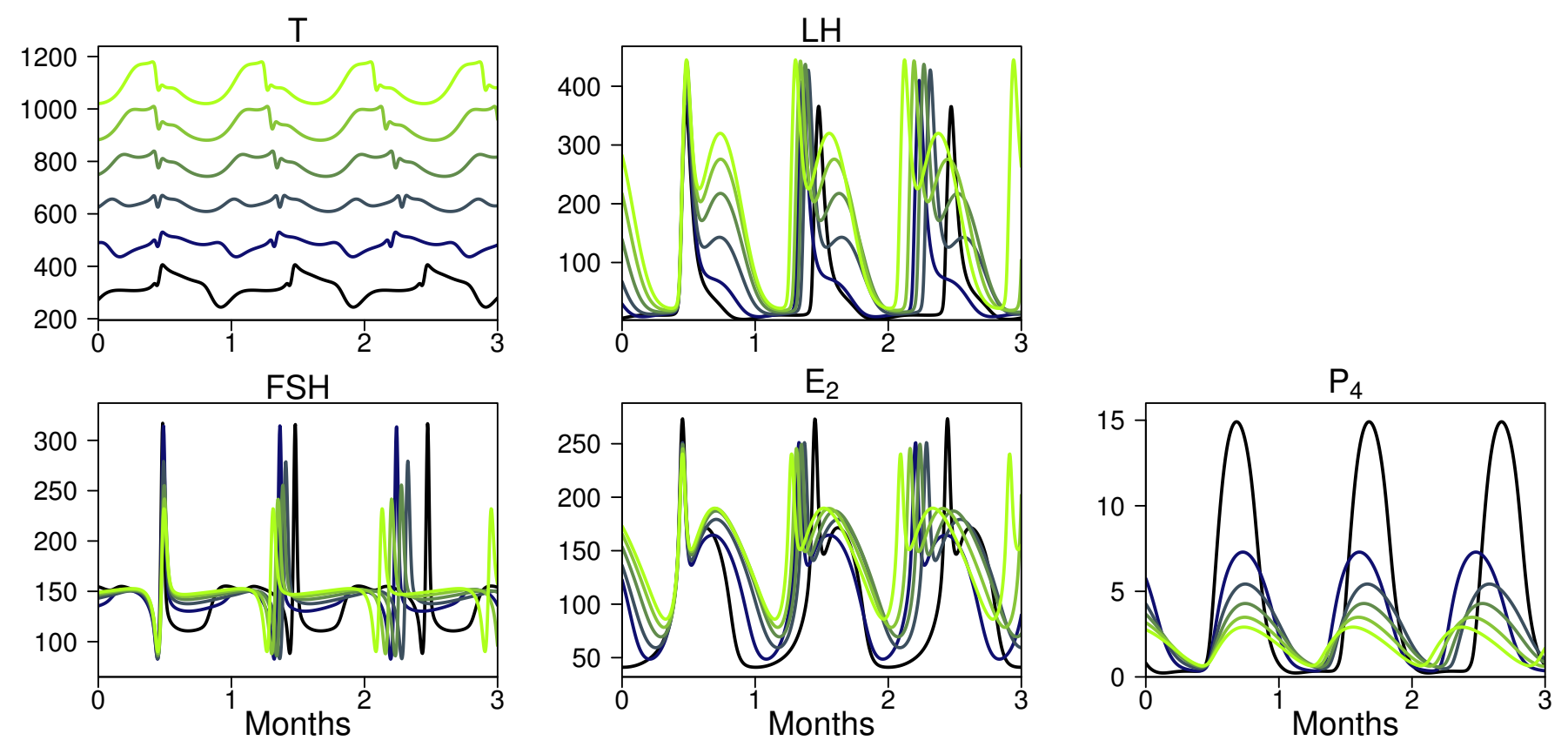

$\alpha=0.00$

$\alpha=1.00 \square \alpha=2.00 \square \alpha=3.00 \square \alpha=4.00$

$\square \alpha=5.00$

Figure 6. Simulated hyperinsulinemia under normal luteinization. Selected trajectories from numerical simulations shown with $\alpha=0,1,2,3,4$, and 5 .
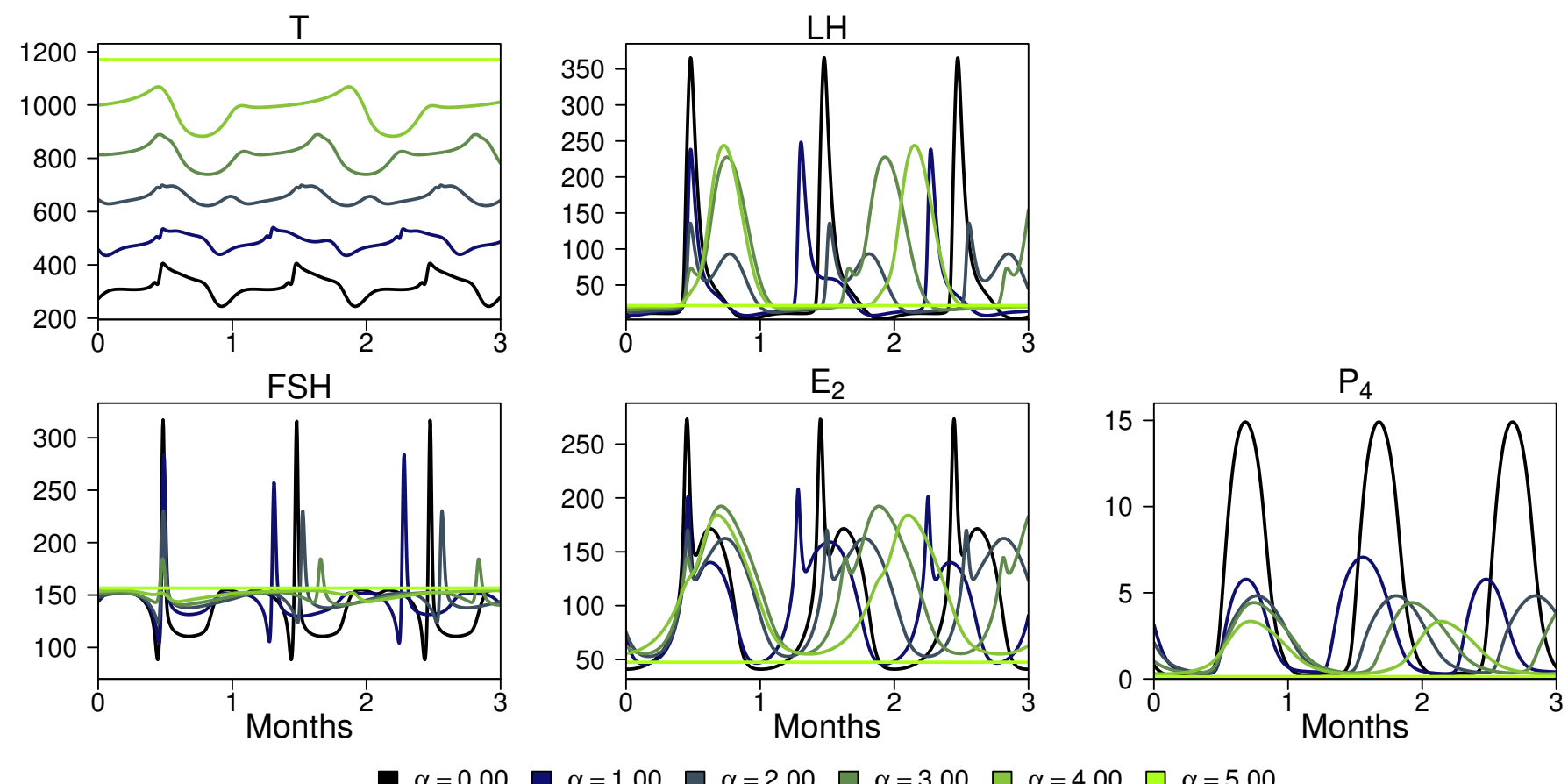

Figure 7. Simulated hyperinsulinemia under premature luteinization. Selected trajectories from numerical simulations shown with $\alpha=$ $0,1,2,3,4$, and 5 . 


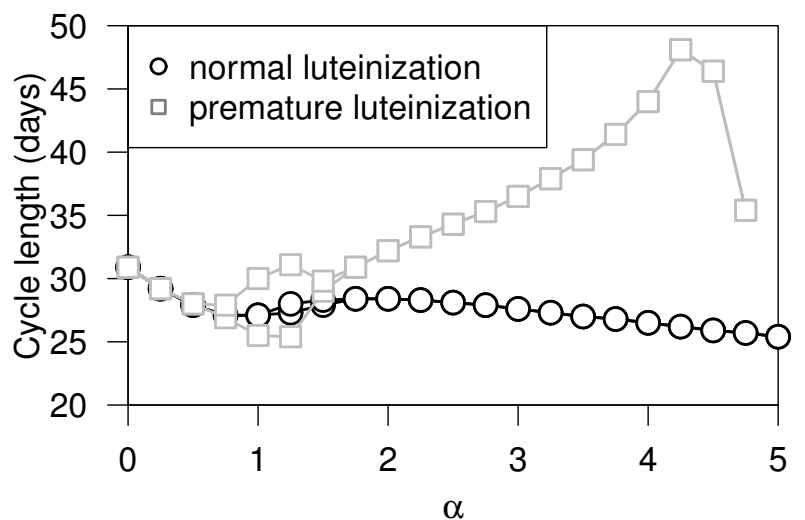

Figure 8. Comparison between normal (NL) and premature (PL) luteinization. Cycle lengths for both models. Two symbols for $\mathrm{a}^{550}$ single value of $\alpha$ indicate lengths for two-cycles. For low $\alpha$, good551 agreement between NL and PL. Model differences increase with $\alpha .{ }_{552}$ NL cycle lengths do not vary significantly, whereas PL lengths increase substantially with intermediate values of $\alpha$. A steady state is ${ }^{553}$ reached for $\alpha=5$, indicated by a missing point.
549

\subsection{Comparison of hyperinsulinemia models}

Here we compare properties of the model under condi- ${ }^{558}$ tions of normal luteinization (NL) and varying levels of $\mathrm{PL}_{560}^{559}$ due to elevated insulin.

Figure 8 compares the effects of PL on cycle length. ${ }_{562}$ With increasing $\alpha$ and NL, the cycle lengths do not vary ${ }_{563}$ substantially, though there is a tendency toward faster $\mathrm{cy}^{-}{ }_{564}$ cles. In contrast, the systemic response to increased insulin ${ }_{565}$ under PL is, for the most part, an elongation of the $\mathrm{cy}^{-}{ }_{566}$ cle. Period-doubling bifurcations have been observed for ${ }_{567}$ related menstrual cycle models [14, 45], and this bifurca- ${ }_{568}$ tion occurs for our model as $\alpha$ changes. We observe cycles $_{569}$ alternating between two cycle lengths for $1 \leqslant \alpha \leqslant 1.5_{570}$ with NL and for $0.75 \leqslant \alpha \leqslant 1.5$ with PL, and these cycle s71 $_{57}$ pairs (two-cycles) correspond to one periodic solution of period approximately 60 days. Oscillations are stable for ${ }_{573}$ all experimental values of $\alpha$ when luteinization is normal. ${ }_{574}$ However, the system approaches a non-oscillatory steady ${ }_{575}$ state for $\alpha=5$ under PL. Further, whereas the LH:FSH ratio steadily inereases with a during $\mathrm{NL}$, the respense of ${ }_{577}$ the ratio under $\mathrm{PL}$ is non-monotonic, with increases for ${ }_{578}$ $2 \leqslant \alpha \leqslant 3.5$ and decreases for $\alpha \geqslant 3.75$ (Figure $8(? ?))$. ${ }_{579}$

Figure 9 a illustrates the values of FSH, LH, E 2 , and ${ }_{580}$ $\mathrm{P}_{4}$ at three isolated points during both NL and PL cycles, for various $\alpha$. We choose the mid-follicular, mid-cycle, and mid-luteal stages because these are important mark- ${ }^{581}$ ers throughout a normal ovulatory cycle. To obtain these 582 quantities, model output is normalized to the fraction of $f_{583}$ an average cycle, with 0 being the start of the cycle and ${ }_{584}$ 1 being the end of a cycle, where applicable. The mid-585 follicular point is assumed to occur at 0.25 , the mid-cycle 586 point at 0.5 , and the mid-luteal point at 0.75 . We are care-587 ful to note that the mid-cycle point does not correspond to588 the preovulatory LH surge. We then determine the value ${ }_{589}$ of the state variables at these points for a given $\alpha$. Since 590 average cycle lengths are used, we may disregard results from cases in which two-cycles occur (shaded regions in Figure 9a.

During NL, increased $\alpha$ has the largest effect on midcycle levels of FSH and mid-luteal levels of LH. The same is true during $\mathrm{PL}$, though there is a more significant decline in mid-cycle FSH. Interestingly, in both cases mid-luteal FSH increases with $\alpha$.

Higher $\alpha$ results in slightly increased mid-cycle LH under NL, but substantially increased mid-luteal LH. This is in stark contrast to the behavior under PL conditions, in which mid-cycle LH monotonically decreases with $\alpha$, and mid-luteal LH increases for $\alpha \leqslant 3.5$ and decreases sharply thereafter.

$\alpha$ also impacts $\mathrm{E}_{2}$ in NL, most notably by raising midollicular $\mathrm{E}_{2}$. There is virtually no difference in mid-cycle $\mathrm{E}_{2}$, whereas mid-luteal $\mathrm{E}_{2}$ increases with $\alpha$. In contrast, during PL, the most drastic effect of $\alpha$ appears to occur during the mid-luteal stage. Mid-cycle $\mathrm{E}_{2}$ also changes significantly, especially for extreme values of $\alpha$ (either near 0 or near 5 ). The impact on mid-follicular $\mathrm{E}_{2}$ is less severe in PL than in NL.

Between the two models, the response of $\mathrm{P}_{4}$ to changes in $\alpha$ are relatively conserved in all stages, where mid-luteal $\mathrm{P}_{4}$ decreases as $\alpha$ increases, and $\mathrm{P}_{4}$ levels at the midfollicular and mid-cycle points are relatively unchanged.

The LH:FSH ratio has often been cited as being abnormal in PCOS. However, the conditions under which this is true vary according to patient characteristics. For example, thin women with PCOS have more often been found to have higher LH:FSH ratios 4, 49. compared to obese women. In addition, LH levels in PCOS have been found to be inversely correlated with body fat percentage 49. Further, the LH:FSH ratio itself exhibits time-dependent changes due to variations in LH and FSH dynamics throughout the course of the cycle. In Figure 9 (b), we observe changes in the LH:FSH ratio relative to the normal cycle (with $\alpha=0$ ) during the mid-follicular, mid-cyle, and mid-luteal points in an average cycle. Under NL, there is a fairly consistent rise with $\alpha$ in the ratio within each phase of the cycle. Under PL, $\alpha$ and the LH:FSH ratio are positively correlated at the mid-follicular time point and negatively correlated at mid-cycle. The changes in the behavior of the ratio at the mid-luteal point is dominated by LH dynamics during both NL and PL.

\subsection{Phenotypes of ovulatory dysfunction}

The Rotterdam criteria for PCOS is the presence of two or more of the following symptoms: hyperandrogenism (HA), anovulation (AO) or oligo-ovulation, and polycystic ovarian morphology (PCO). Given these symptoms, there are four possible forms that PCOS can take in women: one in which all three symptoms are present, and three possible combinations of pairs of symptoms [46, 50, 51. Although the present model cannot account for histological characteristics of the ovaries, we can study the 

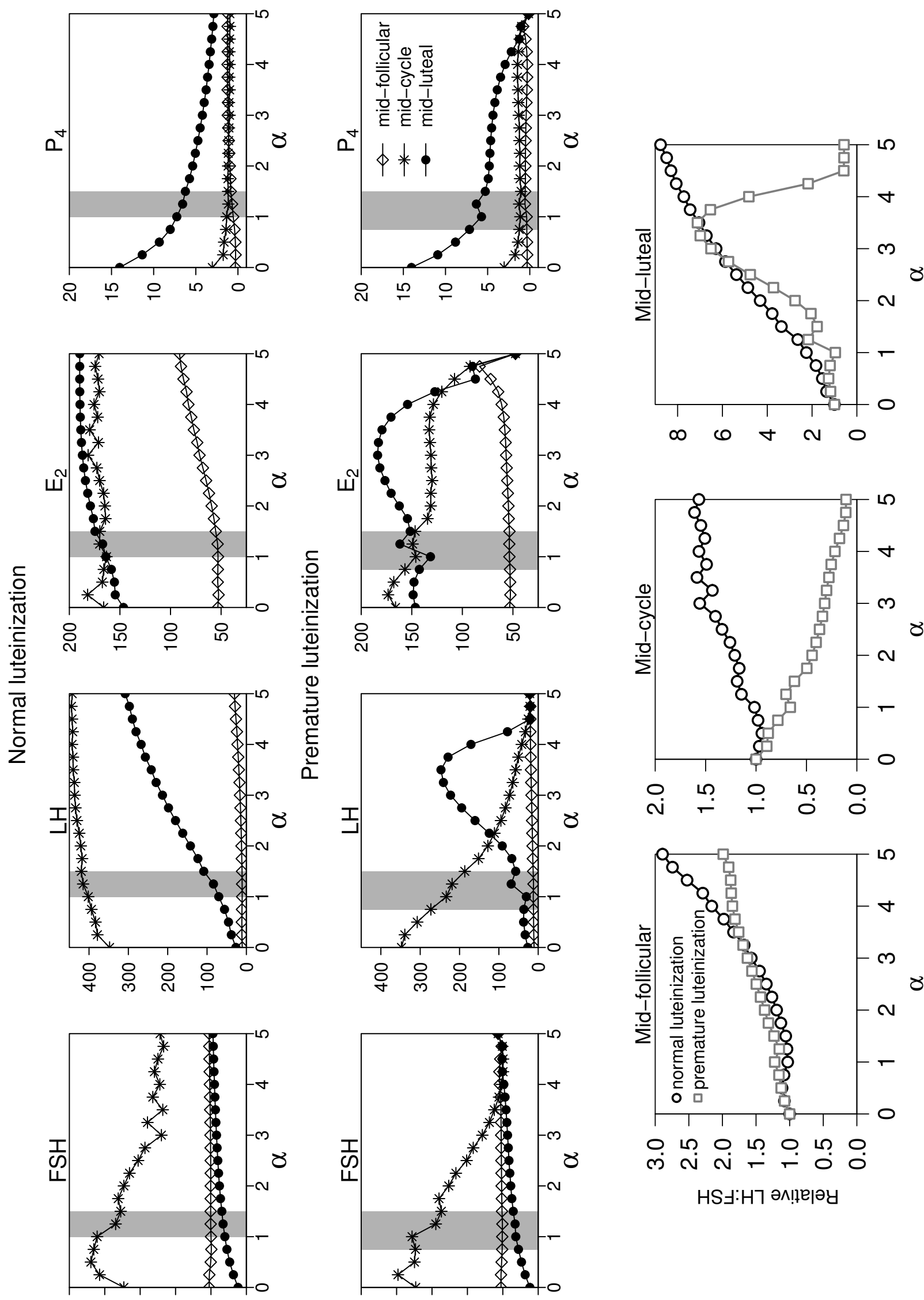

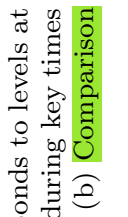

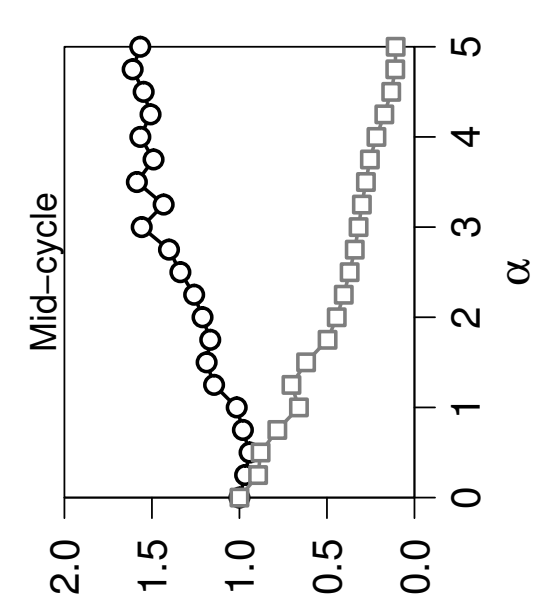

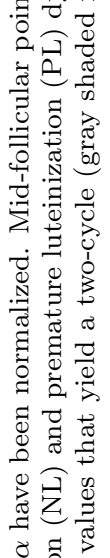

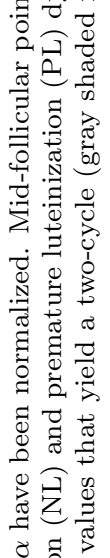

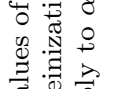

需䨔

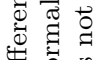

تี

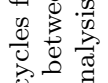

业。

을

ن

[๘]

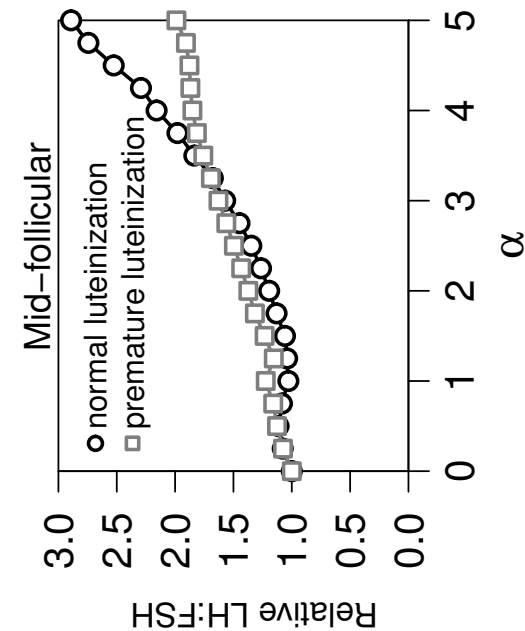

点

类范

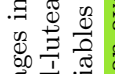

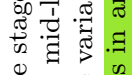

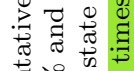

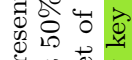

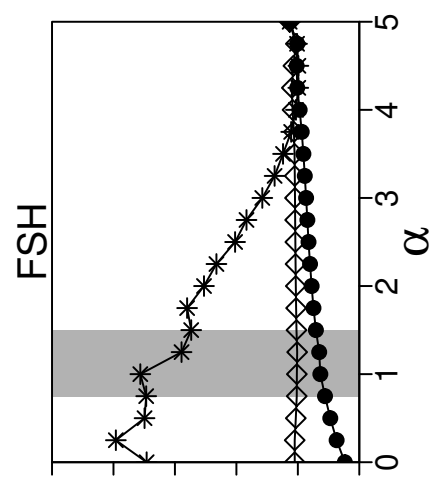

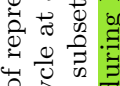

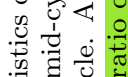

苍式

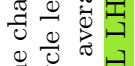

号完卷

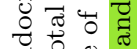

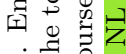

के

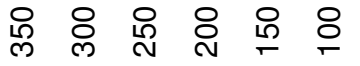

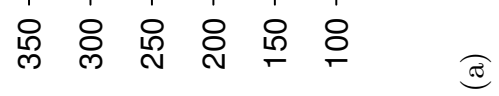

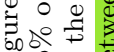


Normal luteinization

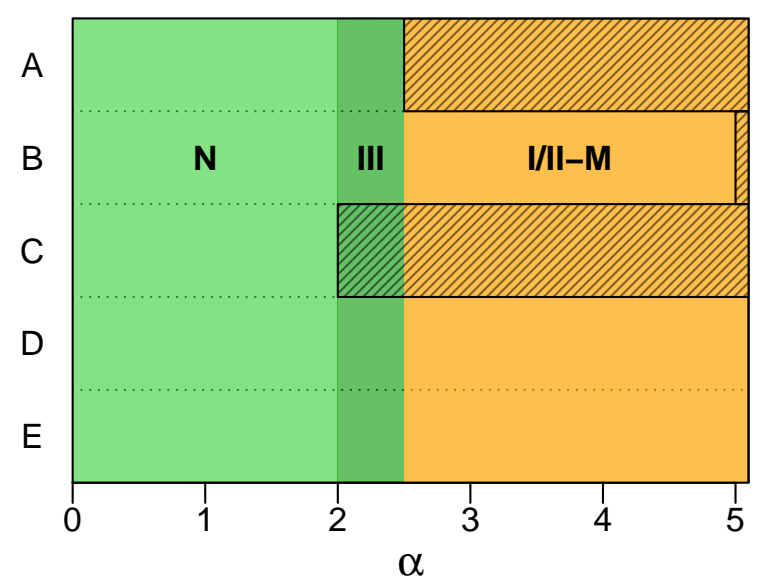

Premature luteinization

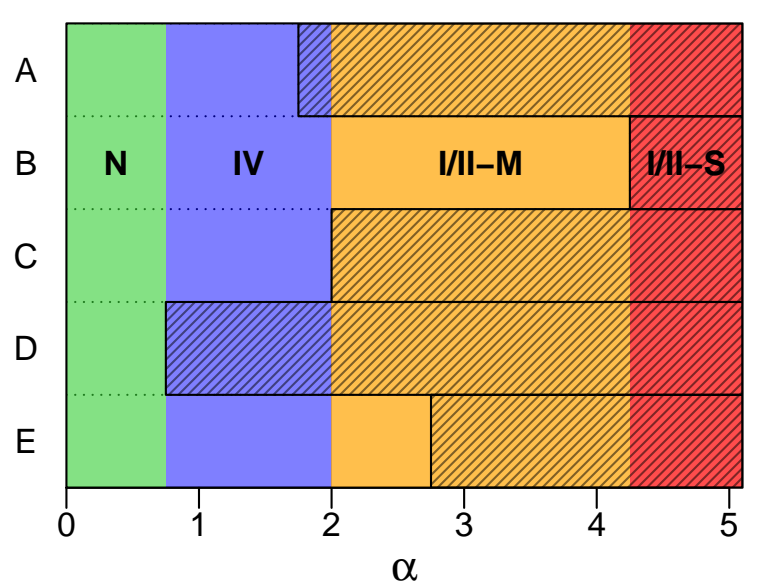

Figure 10. Model summary of characteristic phenotypes for ovulatory dysfunction. Region N: normal ovulatory cycle; Region I/II: ovulatory dysfunction with HA and anovulation; Region III: ovulatory cycle with HA; Region IV: ovulatory dysfunction without HA. The values of $\alpha$ satisfying a criterion are indicated with darker shaded rectangles.

major consequences of hyperinsulinemia and premature 26 luteinization by developing a set of relevant phenotypes627 based on HA and AO alone. We therefore consider three phenotypes to describe generalized ovulatory dysfunction ${ }^{628}$ rather than PCOS. Specifically, we define phenotypes I/II ${ }_{629}$ $\left(\mathrm{HA}^{+} / \mathrm{AO}^{+}\right)$, III $\left(\mathrm{HA}^{+} / \mathrm{AO}^{-}\right)$, and IV $\left(\mathrm{HA}^{-} / \mathrm{AO}^{+}\right)$. Phenotype I/II is so denoted for consistency with phenotypes6з $\mathrm{I}$ and II defined in [51, which are both $\mathrm{HA}^{+} / \mathrm{AO}^{+}$and distinguished by the presence or absence of $\mathrm{PCO}$, respec-631 tively. We use $\mathrm{N}$ to denote the presence of a regular ovulatory cycle without clinical symptoms. Note that phenotype III describes regular ovulatory function in the pres- ${ }_{633}$ ence of HA, and phenotype IV describes ovulatory dys-634 function in the absence of HA.

A specific set of markers determines the levels of insulin-636 mediated feedback required to maintain or disrupt func-637 tion, as well as the severity of dysfunction. We choose 6 бз these markers based on their importance in the menstrual ${ }_{639}$ cycle and PCOS. They are the day $15 \mathrm{LH}$ level $\left(L H_{\alpha}(15)_{, 640}\right.$ after synchronizing the initial cycle for all $\alpha$ to the peak in ${ }_{641}$ $\Omega)$, the maximum $\mathrm{P}_{4}$ concentration $\left(P_{\max }\right)$, and the min-642 imum $\mathrm{T}$ concentration $\left(T_{\min }\right)$. The timing of ovulation is $\mathrm{s}_{643}$ determined by the LH surge. In the absence of a sufficient ${ }_{644}$ surge level, we assume ovulation has not occurred. The $\mathrm{P}_{4645}$ level during the luteal stage is typically a marker confirm- 646 ing ovulation, whereas the LH surge initiates the process.647 A sustained deficiency in $\mathrm{P}_{4}$ can indicate clinical $\mathrm{AO}$ [46].648 We use two markers for AO to identify more closely the 649 presence of ovulatory dysfunction. Finally, because $\mathrm{T}$ and 650 other androgens play a large role in establishing $\mathrm{PCOS}_{, 651}$ we use hyperandrogenism as an additional factor in dys-652 function.

Given these markers, we define a set of threshold quan-654 tities to distinguish between normal and abnormal cycles.655 To allow for mild (M) and severe (S) cases of dysfunction,, 656 we consider two different thresholds for $L H_{\alpha}(15)$ and for $P_{\max }$. The criteria considered are as follows:
A. $P_{\max }<5 \mathrm{ng} / \mathrm{mL}$;
B. $P_{\max }<3 \mathrm{ng} / \mathrm{mL}$ [4];
C. $T_{\min }>600 \mathrm{ng} / \mathrm{L}[46$;
D. $L H_{\alpha}(15)<0.75 \cdot L H_{0}(15)$;
E. $L H_{\alpha}(15)<0.25 \cdot L H_{0}(15)$.

We assume I/II-M denotes the mild I/II phenotype, attained when criterion $\mathrm{C}$, along with either $\mathrm{A}$ or $\mathrm{D}$, is satisfied. Similarly, we assume I/II-S is the severe I/II phenotype, attained when $\mathrm{C}$, along with both $\mathrm{B}$ and $\mathrm{E}$, is satisfied. Further, note that phenotype III is a less severe mode of dysfunction. Phenotype IV, in the absence of HA, we also assume is less severe, as long as neither B or E is satisfied.

In Figure 10, we summarize the results of the NL and PL models to identify the range of $\alpha$ values that satisfy the specified criteria A-E. From left to right, we determine the phenotype of ovulatory dysfunction (regions labeled N, I/II, III, or IV) based on which set of criteria are satisfied for a given $\alpha$ value (patterned rectangles). For example, under PL, there is normal ovulation for $\alpha<0.75$, mild dysfunction with AN but not HA for $0.75 \leqslant \alpha<2$, mild dysfunction with both AN and HA for $2 \leqslant \alpha<4.25$, and a transition to severe dysfunction when $\alpha \geqslant 4.25$.

We find that the presence or absence of premature luteinization determines the likelihood of mild or severe cases of ovulatory dysfunction. Under NL, normal cycles can result for $0 \leqslant \alpha<2$ (region $\mathrm{N}$ ). This window is reduced by more than half in PL. Phenotype III, which is still ovulatory, may occur under NL, but does not with PL. 
Phenotype IV can occur in the presence of PL, but not ${ }_{712}$ with NL. In both cases, hyperandrogenism occurs when 713 $\alpha \geqslant 2$. For the $\alpha$ values tested, higher levels of insulin ${ }_{714}$ feedback are required for dysfunction under NL than under 715 PL. Based on the defined phenotypes, severe dysfunction 716 can only occur under PL. This suggests that PL is not a re-717 quirement for ovulatory dysfunction, but can contribute to718 its severity with less hyperinsulinemia. In addition, mild 719 hyperinsulinemia does not necessarily result in ovulatory 720 dysfunction, and mild hyperandrogenism can still lead to 221 ovulatory cycles as long as PL is not present.

We remark that with the exception of clinically used ${ }_{223}$ thresholds B and C, the distribution of phenotypes is sub-724 ject to our choice of thresholds, primarily for lower val- 725 ues of $\alpha$. For example, if criterion D is reduced to $50 \% 726$ of the normal LH peak, the only change would occur $_{727}$ to regions $\mathrm{N}$ and IV under PL, which would encompass 728 $0 \leqslant \alpha<1.75$ and $1.75 \leqslant \alpha<2$, respectively; the NL pic- 729 ture would remain unchanged (results not shown). Despite 730 threshold variation, the conclusion that PL increases the ${ }_{731}$ severity of dysfunction with less hyperinsulinemia is con-732 sistent.

\section{Discussion}

We have developed a comprehensive mathematical ${ }_{33}$ model of the menstrual cycle with a semi-mechanistic model of ovarian steroid production that incorporates the $з$ з effects of hyperinsulinemia. The model explicitly incorpo-740 rates a role for testosterone $(\mathrm{T})$ in the normal physiological ${ }_{741}$ menstrual cycle, allowing for further exploration in pathol-742 ogy related to ovulatory dysfunction. The model does not 743 explain the origin of hyperinsulinemia, but provides a hy-744 pothesized role for the mechanisms that can lead to hy-745 perandrogenism via elevated insulin. Specifically, an im-746 portant role for insulin is highlighted as a co-gonadotropin ${ }_{747}$ with LH and as a mediator of increased LH sensitivity.748 We do not, however, propose that the only role for insulin 749 is to amplify the effects of LH, as there is likely a clear 750 distinction between mechanisms underlying LH-dependent751 enzymatic processes and cellular differentiation.

Through its description of essential feedback processes 753 that regulate the ovulatory cycle, the model we present754 agrees qualitatively with established clinical data. In ad-755 dition, the model exhibits varying degrees of ovulatory 756 dysfunction depending on the degree of feedback. It also757 allows us to distinguish between less severe types of ovu-758 latory dysfunction based on a particular profile of model 759 output.

Endocrine profiles have been studied previously in761 women with $\mathrm{PCOS}$ [46, 51. In $46, \mathrm{HA}^{+} / \mathrm{AO}^{+} / \mathrm{PCO}^{+}{ }_{762}$ women have increased $\mathrm{LH}$ and LH:FSH ratios, along ${ }_{763}$ with hyperinsulinemia and insulin resistance. Those with 764 $\mathrm{HA}^{+} / \mathrm{AO}^{+} / \mathrm{PCO}^{-}$have many of the same characteristics,765 except that LH levels are statistically significantly higher 760 in the group with $\mathrm{PCO}^{+}$. Panidis and colleagues also de-767 tected higher androgen levels in $\mathrm{HA}^{+} / \mathrm{AO}^{+} / \mathrm{PCO}^{+}$women768 than $\mathrm{HA}^{+} / \mathrm{AO}^{+} / \mathrm{PCO}^{-}$women 51. Phenotype I/II of the current work describes these groups collectively, since the model does not capture physical characteristics of the ovaries. We therefore cannot assess differences in androgen levels within phenotype I/II. The model does support the notion that women with I/II have higher levels of insulin, and hence insulin-mediated feedback, than other individuals with PCOS. This is consistent between both the NL and the PL frameworks.

Based on the endocrine data collected in [46] and [51, it appears that the $\mathrm{HA}^{+} / \mathrm{AO}^{+} / \mathrm{PCO}^{+}$subgroup tends to be more overweight/obese, more insulin-resistant, and have higher $\mathrm{LH}$ levels than the $\mathrm{HA}^{+} / \mathrm{AO}^{+} / \mathrm{PCO}^{-}$subgroup. It is therefore more likely that these individuals are at greater risk for other health complications and may be seen as having a more severe phenotype of PCOS [50. Although our assessment of ovulatory dysfunction phenotypes indicates that lower levels of $L H_{\alpha}(15)$ can result in increased severity, we note that clinical studies measure LH concentrations during the follicular phase of a cycle (roughly days 6-8 of a normal cycle). Follicular phase LH increases in both the PL and NL models (see Figure 9a), though to a greater extent under NL. Our results are therefore consistent with these reports.

Also consistent with studies of women with PCOS is phenotype III. Women with ovulatory PCOS have been found to have all of the metabolic and endocrine markers of PCOS, but in a less extreme form [46. Testosterone, though elevated, seemed to fall between the level in the control group and those with $\mathrm{HA}^{+} / \mathrm{AO}^{+} / \mathrm{PCO}^{+}$. In [51, no differences between insulin resistance markers were found in the ovulatory PCOS group than control individuals. This is in contrast to the present model, which indicates a slight increase in feedback from hyperinsulinemia. Nevertheless, it is clear that this group of individuals is closest to normo-ovulatory.

Normoandrogenic women with PCOS may or may not be more insulin resistant than normally ovulating women 51. However, ovulatory dysfunction is evident even in the absence of HA. The present model produces these results, with the distinction being determined by the presence of PL. For phenotype IV, the range of insulin resistance, as indicated by insulin-mediated feedback strength $\alpha$, is higher than normal in the model with PL, but may be higher than or similar to levels in the NL version of the model. Interestingly, there is much debate on whether normoandrogenic PCOS is simply an anomaly that should not be classified beneath the PCOS umbrella. This is certainly possible within the current framework, if we consider the nature of the "mild" endocrine markers used to summarize phenotypes. Reducing the $L H_{\alpha}(15)$ threshold, which is already arbitrary, would virtually eliminate phenotype IV completely. In this case, there would be no obvious differences between women with NL and those with PL.

Collectively, our results indicate that as long as PL is present, any abnormalities to the cycles result in ovulatory dysfunction. This is in contrast to NL, in which normal 
ovulation can persist in the face of HA. This has been 824 observed previously, where prematurely luteinized follicu-825 lar cells come only from women with PCOS who are also anovulatory, but none who are ovulatory 48 .

Many studies of women with PCOS track LH and $\mathrm{FSH}^{82}$ levels only during the follicular stage of a cycle [46, 50, 51]. ${ }_{827}$ This approach does not lend itself to comparison with the ${ }_{828}$ full cycle information provided by the model herein. $\mathrm{Al}_{829}^{20}$ though we see an increase in the LH:FSH ratio during the follicular phase of both NL and PL, we see drastically different results after examining the LH:FSH ratio during the remainder of the cycle (see Figure 9). In particular, we are interested in the consistent decline in mid-cycle LH during PL, which appears to account for much of the difference. An interesting factor to consider is that in both NL and $\mathrm{PL}$, the $\Phi$-to- $\Omega$ transition is accelerated, resulting in reduced follicle growth. In NL, this is due to increased LH; in PL, it is due to premature luteinization. Therefore, the question is why does PL allow for a reduction in the $\mathrm{LH}$ surge level with increasing $T$ levels when NL does not. The answer lies in the fact that if follicles are more sensitive to LH earlier in their development, it will take less LH to initiate a progression to the (mathematical) ovulatory phase. As the insulin-mediated feedback increases, less and less LH is required, until the LH surge is absent. Even in these instances, there is a progression through the remaining ovulatory stages of the model. This is unlikely to be the case physiologically, as ovulation would simply not occur at all. One future modification is to separate the processes of luteinization and ovulation. This will effectively incorporate the role of LH as a growth hormone during the late follicular stage. Whether NL or PL is present, the model results suggest that increased LH activity in the ovaries is a significant contributor to ovulatory dysfunction.

An important influence of hyperandrogenism on the disrupted menstrual cycle is, according to the present work, largely dependent on feedback between T and LH. By incorporating a normal physiological role for $\mathrm{T}$ in the cycle and by modeling $\mathrm{T}$ production in consideration of the underlying biological processes, we are able to observe abnormalities in the system under perturbation of key biological processes. The inclusion of luteinization as a factor underlying androgen-mediated dysfunction also reveals a cause of increased hyperandrogenism when insulinmediated feedback is excessively high. Collectively, the present model gives insight into the mechanisms leading to ovulatory dysfunction once hyperinsulinemia is in place. The precise role for insulin, however, must be further elucidated, as its function in the current model is as a proposed instigator of hyperandrogenism without the associated mechanisms.

\section{Acknowledgements}

Special thanks to Claude Hughes for helpful conversations. This work was supported by the National Science Foundation [DMS-1225607] (J.F.S.) and The NSF Alliance for Building Faculty Diversity in the Mathematical Sciences [DMS-0946431] (E.J.G.).

\section{AppendixA. Model Parameters}

Table A.1 lists the parameters used for model simulation, along with the initial conditions of all state variables. 
Table A.1. All model parameters. Dimensionless quantities are denoted by \#. * denotes fixed (non-estimated) parameters. $\dagger$ denotes derived parameters (see text for details). Note: $m$ and $\tau_{2}$ are fixed arbitrarily based on model assumptions.

(a) Parameter values used in the pituitary subsystem.

\begin{tabular}{lcl}
\hline Parameter & Units & Value \\
\hline \hline$c_{F, E}$ & $(\mathrm{ng} / \mathrm{L})^{-2}$ & $2.2729 \times 10^{-3}$ \\
$c_{F, I}$ & $\#$ & 1.9488 \\
$c_{F, P}$ & $(\mu \mathrm{g} / \mathrm{L})^{-1}$ & 60.428 \\
$c_{L, E}$ & $(\mathrm{ng} / \mathrm{L})^{-1}$ & $1.0404 \times 10^{-3}$ \\
$c_{L, P}$ & $(\mu \mathrm{g} / \mathrm{L})^{-1}$ & $9.9415 \times 10^{-3}$ \\
$c_{L, T}$ & $(\mathrm{ng} / \mathrm{L})^{-1}$ & $9.5942 \times 10^{-3}$ \\
$\delta_{F}$ & $1 / \mathrm{d}$ & $8.21^{*}[13]$ \\
$\delta_{L}$ & $1 / \mathrm{d}$ & $14^{*}[13]$ \\
$k_{F}$ & $1 / \mathrm{d}$ & 2.5412 \\
$K_{F, I}$ & $\mu \mathrm{g}$ & 107.01 \\
$K_{i L, P}$ & $\mu \mathrm{g} / \mathrm{L}$ & 0.34952 \\
$K_{L, T}$ & $\mathrm{ng} / \mathrm{L}$ & $420^{*}[17]$ \\
$K_{m L}$ & $\mu \mathrm{g} / \mathrm{L}$ & 183.56 \\
$k_{L}$ & $1 / \mathrm{d}$ & 0.74567 \\
$n$ & $\#$ & $8^{*}[13]$ \\
$V$ & 1 & $2.5^{*}[13$ \\
$v_{0 L}$ & $\mu \mathrm{g} / \mathrm{d}$ & 1051.7 \\
$v_{1 L}$ & $\mu \mathrm{g} / \mathrm{d}$ & 34838 \\
$v_{F}$ & $\mu \mathrm{g} / \mathrm{d}$ & 3236.6
\end{tabular}

(c) Parameter values used in the steroidogenesis subsystem.

\begin{tabular}{lcl}
\hline \multicolumn{1}{c}{ Parameter } & Units & Value \\
\hline \hline$c_{T, F_{2}}$ & $\mu \mathrm{g} / \mathrm{L}$ & $123.8136^{\dagger}$ \\
$\delta_{E}$ & $1 / \mathrm{d}$ & $1.1^{*}[52,53]$ \\
$\delta_{P},[5]$ \\
$\delta_{T}$ & $1 / \mathrm{d}$ & $0.5^{*}[5]$ \\
$e_{0}$ & $1 / \mathrm{d}$ & $5.5^{*}[55]$ \\
$\eta$ & $\mathrm{ng} / \mathrm{L} \cdot \mathrm{d}$ & 44.512 \\
$h_{3}$ & $\#$ & 1.1087 \\
$\kappa_{1}$ & $\mu \mathrm{g} / \mathrm{L}$ & 17.796 \\
$\kappa_{2}$ & $\#$ & 1.09 \\
$\kappa_{3}$ & $\mu \mathrm{g} / \mathrm{L}$ & 22.28645 \\
$p$ & $(\mu \mathrm{g} / \mathrm{L})^{2}$ & $113.9188^{\dagger}$ \\
$t_{0}$ & $1 / \mathrm{L} \cdot \mathrm{d}$ & 0.3734 \\
$t_{1}$ & $\mathrm{ng} / \mathrm{L}$ & 741.68 \\
$t_{2}$ & $\mathrm{ng} / \mathrm{L} \cdot \mu \mathrm{g} \cdot \mathrm{d}$ & 0.57088 \\
$\tau_{1}$ & $\mathrm{ng} / \mathrm{L} \cdot \mu \mathrm{g} \cdot \mathrm{d}$ & 1.3481 \\
$\tau_{2}$ & $\#$ & 5.3989 \\
$\tau_{3}$ & $\#$ & $0^{*}$ \\
$t_{g 1}$ & $\mu \mathrm{g}$ & 430.91 \\
$t_{g 2}$ & $\mathrm{ng} / \mathrm{L} \cdot \mu \mathrm{g} \cdot \mathrm{d}$ & 6.6548 \\
$\Psi$ & $1 / \mathrm{d}$ & 186.27 \\
& $\mu \mathrm{g}$ & 2004.3
\end{tabular}

(b) Parameter values used in the follicular dynamics subsystem.

\begin{tabular}{lcl}
\hline \multicolumn{1}{c}{ Parameter } & Units & Value \\
\hline \hline$c_{\Phi, F}$ & $(\mu \mathrm{g} / \mathrm{L})^{-1}$ & 0.01127 \\
$\delta_{s}$ & $1 / \mathrm{d}$ & 0.74702 \\
$f_{0}$ & $\mu \mathrm{g} / \mathrm{d}$ & $2.5112 \times 10^{-3}$ \\
$f_{1}$ & $1 / \mathrm{d}$ & 4.3764 \\
$f_{2}$ & $1 / \mathrm{d}$ & 27.812 \\
$h_{1}$ & $\mu \mathrm{g} / \mathrm{L}$ & 590.32 \\
$h_{2}$ & $\mu \mathrm{g} / \mathrm{L}$ & 1815.3 \\
$h_{p}$ & $\mu \mathrm{g} / \mathrm{L}$ & 20.764 \\
$h_{s}$ & $\mu \mathrm{g} / \mathrm{L}$ & 12.329 \\
$l$ & $1 / \mathrm{d}$ & 0.49017 \\
$m$ & $\#$ & 4 \\
$\hat{s}$ & $1 / \mathrm{d}$ & 2.378 \\
$w$ & $1 / \mathrm{d}$ & 0.23173
\end{tabular}

(d) Initial conditions used in the physiological model.

\begin{tabular}{ccc}
\hline Variable & Units & Initial Value \\
\hline \hline$F S H$ & $\mu \mathrm{g} / \mathrm{L}$ & 142.5 \\
$L H$ & $\mu \mathrm{g} / \mathrm{L}$ & 25.34 \\
$F S H_{\rho}$ & $\mu \mathrm{g}$ & 116.82 \\
$L H_{\rho}$ & $\mu \mathrm{g}$ & 250.35 \\
$\Phi$ & $\mu \mathrm{g}$ & 0.50185 \\
$\Omega$ & $\mu \mathrm{g}$ & 9.7509 \\
$\Lambda$ & $\mu \mathrm{g}$ & 4.102 \\
$S$ & $\#$ & 0.050498 \\
$T_{\gamma}$ & $\mathrm{ng} / \mathrm{L} \cdot \mu \mathrm{g}$ & 0.003999 \\
$T$ & $\mathrm{ng} / \mathrm{L}$ & 273.67 \\
$E_{2}$ & $\mathrm{ng} / \mathrm{L}$ & 56.387 \\
$P_{4}$ & $\mathrm{ng} / \mathrm{mL}$ & 0.468
\end{tabular}




\section{References}

[1] C. G. Baptiste, M.-C. Battista, A. Trottier, J.-P. Baillargeon, Insulin and hyperandrogenism in women with polycystic ovary ${ }_{904}$ syndrome, The Journal of steroid biochemistry and molecular ${ }_{905}$ biology 122 (2010) 42-52.

[2] S. Jonard, D. Dewailly, The follicular excess in polycystic ${ }_{900}^{900}$ ovaries, due to intra-ovarian hyperandrogenism, may be the ${ }_{908}$ main culprit for the follicular arrest, Human Reproduction Up- ${ }_{909}$ date 10 (2004) 107-117.

[3] H. Mason, A. Colao, U. Blume-Peytavi, S. Rice, A. Qureshi, ${ }_{911}^{910}$ L. Pellatt, F. Orio, S. L. Atkin, Polycystic ovary syndrome ${ }_{912}$ (PCOS) trilogy: a translational and clinical review, Clinical ${ }_{913}$ endocrinology 69 (2008) 831-844

[4] M. O. Goodarzi, D. A. Dumesic, G. Chazenbalk, R. Azziz, Poly- ${ }_{915}$ cystic ovary syndrome: etiology, pathogenesis and diagnosis, ${ }_{916}$ Nature Reviews Endocrinology 7 (2011) 219-231.

[5] B. Bhagavath, W. Vitek, J. Queenan, K. Hoeger, Metformin ${ }_{917}^{917}$ and other insulin sensitizers in polycystic ovary syndrome, in: ${ }_{919}$ Seminars in reproductive medicine, volume 32 , Thieme Medical $_{920}$ Publishers, 2014, pp. 323-330.

[6] M. F. Costello, J. A. Eden, A systematic review of the repro- ${ }_{922}$ ductive system effects of metformin in patients with polycystic ${ }_{923}$ ovary syndrome, Fertility and sterility 79 (2003) 1-13.

[7] D. J. Bernard, J. Fortin, Y. Wang, P. Lamba, Mechanisms of ${ }_{925}^{924}$ FSH synthesis: What we know, what we don't, and why you ${ }_{926}$ should care, Fertility and sterility 93 (2010) 2465-2485.

[8] N. C. Buffet, C. Djakoure, S. C. Maitre, P. Bouchard, Regulation of the human menstrual cycle, Frontiers in neuroen- ${ }_{929}$ docrinology 19 (1998) 151-186.

[9] A. R. Genazzani, F. Petraglia, O. Gamba, L. Sgarbi, M. Greco, ${ }_{93}$ A. D. Genazzani, Neuroendocrinology of the menstrual cycle, ${ }_{932}$ Annals of the New York Academy of Sciences 816 (1997) 143- ${ }_{933}$ 150.

[10] C. C. Keefe, M. M. Goldman, K. Zhang, N. Clarke, R. E. Reitz, ${ }_{935}$ C. K. Welt, Simultaneous measurement of thirteen steroid hor- ${ }_{936}$ mones in women with polycystic ovary syndrome and control ${ }_{937}$ women using liquid chromatography-tandem mass spectrome- ${ }_{938}$ try, PloS one 9 (2014) e93805.

[11] I. Sinha-Hikim, S. Arver, G. Beall, R. Shen, M. Guerrero, F. Sattler, C. Shikuma, J. C. Nelson, B.-M. Landgren, N. A.944 Mazer, et al., The use of a sensitive equilibrium dialysis method ${ }_{942}$ for the measurement of free testosterone levels in healthy, cy- ${ }_{943}$ cling women and in human immunodeficiency virus-infected ${ }_{944}$ women 1, The Journal of Clinical Endocrinology \& Metabolism $_{945}$ 83 (1998) 1312-1318.

[12] P. M. Schlosser, J. F. Selgrade, A model of gonadotropin regula- ${ }_{947}^{946}$ tion during the menstrual cycle in women: Qualitative features, Environmental health perspectives (2000) 873-881.

13] L. H. Clark, P. M. Schlosser, J. F. Selgrade, Multiple stable pe- ${ }_{950}$ riodic solutions in a model for hormonal control of the menstrual cycle, Bulletin of mathematical biology 65 (2003) 157-173.

[14] A. O. Hendrix, C. L. Hughes, J. F. Selgrade, Modeling endocrine control of the pituitary-ovarian axis: Androgenic influence and ${ }_{954}$ chaotic dynamics, Bulletin of mathematical biology $76(2014)_{955}^{954}$ $136-156$.

[15] C. Chen, J. P. Ward, A mathematical model for the human ${ }_{95}^{956}$ menstrual cycle, Mathematical Medicine and Biology 31 (2014) 958 $_{95}$ $65-86$.

16] C. Welt, Y. Sidis, H. Keutmann, A. Schneyer, Activins, inhibins, and follistatins: From endocrinology to signaling. $A_{961}$ paradigm for the new millennium, Experimental Biology and ${ }_{962}$ Medicine 227 (2002) 724-752.

[17] M. Yasin, A. C. Dalkin, D. J. Haisenleder, J. C. Marshall, ${ }_{964}^{963}$ Testosterone is required for gonadotropin-releasing hormone ${ }_{965}$ stimulation of luteinizing hormone- $\beta$ messenger ribonucleic $\operatorname{acid}_{966}$ expression in female rats., Endocrinology 137 (1996) 1265-1271. ${ }_{967}$

[18] M. G. Ropelato, M. C. Garcia Rudaz, M. E. Escobar, S. V.968 Bengolea, M. L. Calcagno, J. D. Veldhuis, M. Barontini, Acute ${ }_{969}$ effects of testosterone infusion on the serum luteinizing hormone ${ }_{970}$ profile in eumenorrheic and polycystic ovary syndrome adoles- cents, The Journal of Clinical Endocrinology \& Metabolism 94 (2009) 3602-3610

19] S. Blank, C. McCartney, J. Marshall, The origins and sequelae of abnormal neuroendocrine function in polycystic ovary syndrome, Human Reproduction Update 12 (2006) 351-361.

[20] L. Pal, Polycystic Ovary Syndrome: Current and Emerging Concepts, Springer Science \& Business Media, 2014.

21] A. Gougeon, Dynamics of follicular growth in the human: A model from preliminary results, Human Reproduction 1 (1986) 81-87.

[22] A. R. Baerwald, G. P. Adams, R. A. Pierson, Ovarian antral folliculogenesis during the human menstrual cycle: A review, Human reproduction update 18 (2012) 73-91.

[23] G. D. Niswender, J. L. Juengel, P. J. Silva, M. K. Rollyson, E. W. McIntush, Mechanisms controlling the function and life span of the corpus luteum, Physiological reviews 80 (2000) 1-29.

[24] C. G. Gervásio, M. P. Bernuci, M. F. Silva-de Sá, A. C. J. d. S. Rosa-e Silva, The role of androgen hormones in early follicular development, ISRN obstetrics and gynecology 2014 (2014).

[25] A. E. Drummond, The role of steroids in follicular growth, Reprod Biol Endocrinol 4 (2006) 450.

[26] H. Prizant, N. Gleicher, A. Sen, Androgen actions in the ovary: balance is key, Journal of Endocrinology 222 (2014) R141-R151.

27] A. Sen, H. Prizant, A. Light, A. Biswas, E. Hayes, H.-J. Lee, D. Barad, N. Gleicher, S. R. Hammes, Androgens regulate ovarian follicular development by increasing follicle stimulating hormone receptor and microRNA-125b expression, Proceedings of the National Academy of Sciences 111 (2014) 3008-3013.

[28] G. Erickson, C. Wang, A. Hsueh, FSH induction of functional LH receptors in granulosa cells cultured in a chemically defined medium, Nature 279 (1979).

[29] S. G. Hillier, M. Tetsuka, Role of androgens in follicle maturation and atresia, Baillière's clinical obstetrics and gynaecology 11 (1997) 249-260.

[30] M. Gibson, Corpus luteum, Glob. Libr. Women's Medicine (2008).

[31] R. L. Stouffer, C. V. Bishop, R. L. Bogan, F. Xu, J. D. Hennebold, Endocrine and local control of the primate corpus luteum, Reproductive biology 13 (2013) 259-271.

[32] L. Devoto, A. Fuentes, P. Kohen, P. Céspedes, A. Palomino, R. Pommer, A. Muñoz, J. F. Strauss, The human corpus luteum: life cycle and function in natural cycles, Fertility and sterility 92 (2009) 1067-1079.

[33] L. Poretsky, N. A. Cataldo, Z. Rosenwaks, L. C. Giudice, The insulin-related ovarian regulatory system in health and disease, Endocrine reviews 20 (1999) 535-582.

[34] J. K. Wickenheisser, J. M. McAllister, Ovarian steroidogenic abnormalities in PCOS, in: The Polycystic Ovary Syndrome: Current Concepts On Pathogenesis And Clinical Care, Springer, 2007, pp. 69-84.

[35] J.-P. Baillargeon, Insulin action in polycystic ovary syndrome: in vivo and in vitro, in: The Polycystic Ovary Syndrome: Current Concepts On Pathogenesis And Clinical Care, Springer, 2007, pp. 43-68.

[36] D. Cadagan, R. Khan, S. Amer, Thecal cell sensitivity to luteinizing hormone and insulin in polycystic ovarian syndrome, Reproductive Biology 16 (2016) 53-60.

37] C. M. B. Solorzano, J. P. Beller, M. Y. Abshire, J. S. Collins, C. R. McCartney, J. C. Marshall, Neuroendocrine dysfunction in polycystic ovary syndrome, Steroids 77 (2012) 332-337.

[38] J. Young, A. S. McNeilly, Theca: the forgotten cell of the ovarian follicle, Reproduction 140 (2010) 489-504.

[39] R. Horton, J. Tait, Androstenedione production and interconversion rates measured in peripheral blood and studies on the possible site of its conversion to testosterone., Journal of Clinical Investigation 45 (1966) 301.

[40] I. E. Messinis, Ovarian feedback, mechanism of action and possible clinical implications, Human reproduction update 12 (2006) $557-571$.

[41] N. Doldi, A. Gessi, A. Destefani, F. Calzi, A. Ferrari, Polycystic ovary syndrome: anomalies in progesterone production, Human 
Reproduction 13 (1998) 290-293

[42] R. I. McLachlan, N. L. Cohen, K. D. Dahl, W. J. Bremner, M. R. Soules, Serum inhibin levels during the periovulatory interval in normal women: relationships with sex steroid and gonadotrophin levels, Clinical endocrinology 32 (1990) 39-48.

[43] A. Sanchez, digitize, MATLAB Central File Exchange, http://www .mathworks .com/matlabcentral/fileexchange/ 8139-digitize 2005. Retrieved: September 18, 2013.

[44] A. Margolskee, J. F. Selgrade, Dynamics and bifurcation of a model for hormonal control of the menstrual cycle with inhibin delay, Mathematical biosciences 234 (2011) 95-107.

[45] N. M. Panza, A. A. Wright, J. F. Selgrade, A delay differential equation model of follicle waves in women, Journal of biological dynamics 10 (2016) 200-221.

[46] E. Guastella, R. A. Longo, E. Carmina, Clinical and endocrine characteristics of the main polycystic ovary syndrome phenotypes, Fertility and sterility 94 (2010) 2197-2201.

[47] R. Anderson, N. Groome, D. Baird, Inhibin A and inhibin B in women with polycystic ovarian syndrome during treatment with FSH to induce mono-ovulation, Clinical endocrinology 48 (1998) 577-584.

[48] D. S. Willis, H. Watson, H. D. Mason, R. Galea, M. Brincat, S. Franks, Premature response to luteinizing hormone of granulosa cells from anovulatory women with polycystic ovary syndrome: Relevance to mechanism of anovulation, The Journal of Clinical Endocrinology \& Metabolism 83 (1998) 3984-3991.

[49] A. E. Taylor, B. McCourt, K. A. Martin, E. J. Anderson, J. M. Adams, D. Schoenfeld, J. E. Hall, Determinants of abnormal gonadotropin secretion in clinically defined women with polycystic ovary syndrome 1, The Journal of Clinical Endocrinology \& Metabolism 82 (1997) 2248-2256.

[50] C. Welt, J. Gudmundsson, G. Arason, J. Adams, H. Palsdottir, G. Gudlaugsdottir, G. Ingadottir, W. Crowley, Characterizing discrete subsets of polycystic ovary syndrome as defined by the Rotterdam criteria: The impact of weight on phenotype and metabolic features, The Journal of Clinical Endocrinology \& Metabolism 91 (2006) 4842-4848.

[51] D. Panidis, K. Tziomalos, G. Misichronis, E. Papadakis, G. Betsas, I. Katsikis, D. Macut, Insulin resistance and endocrine characteristics of the different phenotypes of polycystic ovary syndrome: A prospective study, Human reproduction 27 (2012) 541-549.

[52] National Center for Biotechnology Information, Pubchem compound database; $\mathrm{CID}=5757$, https://pubchem.ncbi.nlm.nih. gov/compound/5757 2016. Retrieved: December 2, 2016.

[53] E. S. Ginsburg, X. Gao, B. F. Shea, R. L. Barbieri, Half-life of estradiol in postmenopausal women, Gynecologic and obstetric investigation 45 (1998) 45-48.

[54] National Center for Biotechnology Information, Pubchem compound database; $\mathrm{CID}=5994$, https://pubchem.ncbi.nlm.nih. gov/compound/5994 2016. Retrieved: December 2, 2016.

[55] National Center for Biotechnology Information, Pubchem compound database; CID =5994, https://pubchem.ncbi.nlm.nih. gov/compound/6013 2016. Retrieved: December 2, 2016.

[56] N. S. Houwing, F. Maris, P. G. Schnabel, W. M. Bagchus, Pharmacokinetic study in women of three different doses of a new formulation of oral testosterone undecanoate, Andriol Testocaps, Pharmacotherapy: The Journal of Human Pharmacology and Drug Therapy 23 (2003) 1257-1265. 